ANL-6701

Reactor Technology (TID-4500, 20th Ed.) AEC Research and Development Report

ARGONNE NATIONAL LABORATORY

9700 South Cass Avenue

Argonne, Illinois

\title{
BASIC NUCLEAR AND REACTOR INFORMATION FOR THE REACTOR OPERATOR
}

Edited by

E. G. Greenman

Reactor Operations Division

February 1963

Operated by The University of Chicago under

Contract W-31-109-eng-38 with the

U. S. Atomic Energy Commission 


\section{DISCLAIMER}

This report was prepared as an account of work sponsored by an agency of the United States Government. Neither the United States Government nor any agency Thereof, nor any of their employees, makes any warranty, express or implied, or assumes any legal liability or responsibility for the accuracy, completeness, or usefulness of any information, apparatus, product, or process disclosed, or represents that its use would not infringe privately owned rights. Reference herein to any specific commercial product, process, or service by trade name, trademark, manufacturer, or otherwise does not necessarily constitute or imply its endorsement, recommendation, or favoring by the United States Government or any agency thereof. The views and opinions of authors expressed herein do not necessarily state or reflect those of the United States Government or any agency thereof. 


\section{DISCLAIMER}

Portions of this document may be illegible in electronic image products. Images are produced from the best available original document. 


\section{FOREWORD}

The information contained in this book is essentially that presented in a series of lectures given in August 1961. Those lectures were for the purpose of giving Junior Reactor Operators a qualitative understanding of reactors. Mr. E. G. Greenman attended these lectures, as a student, and subsequently undertook the task of transforming his lecture notes into this book. He was assisted in this effort by his colleague, Mr. W. O. Flurkey.

Staff members of the Reactor Operations Division who prepared the original series of lectures are: J. A. Beidelman, J.H. Norman, E. R. Stansberry and J.H. Talboy. 
TABLE OF CONTENTS

Page

INTRODUCTION.

I. THE FISSION PROCESS AND THE CHAIN REACTION. ....

1.0 Spontaneous and Induced Fission............ 11

1.1 The Moderation Process............... 11

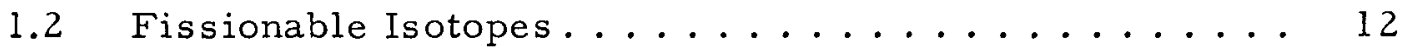

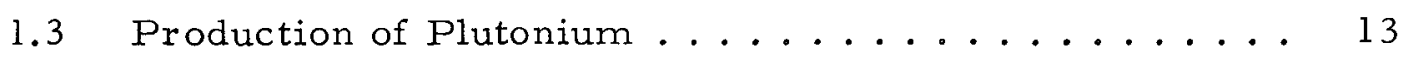

1.4 Fission Yield of Uranium-235............. 14

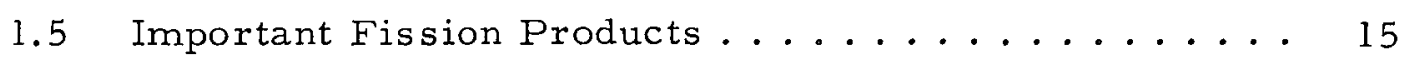

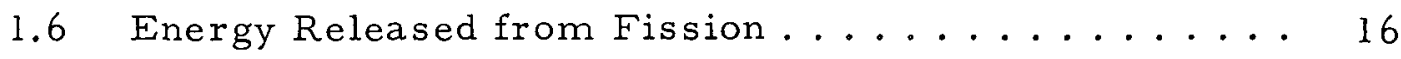

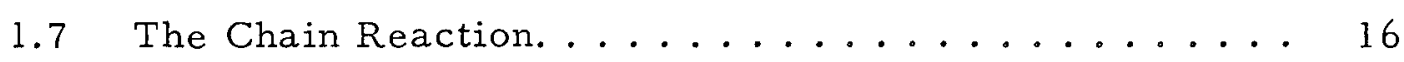

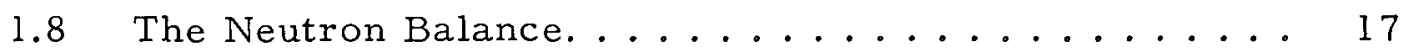

1.9 Subcritical Multiplication.............. 17

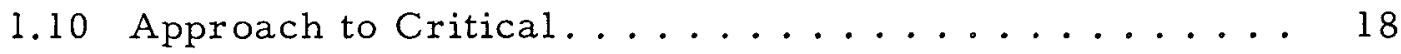

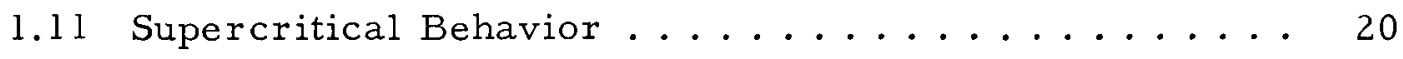

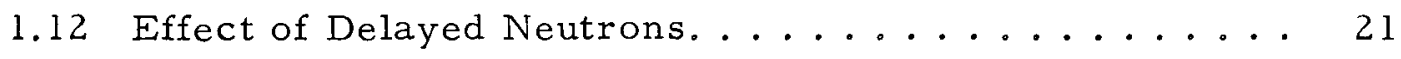

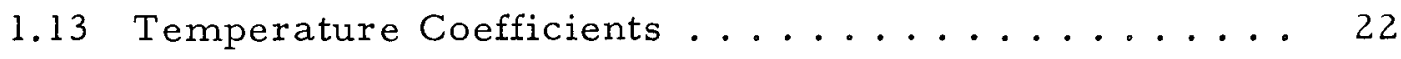

II. REACTOR STRUCTURAL COMPONENTS........... 23

2.0 Structural Form Versus Neutron Balance ....... 23

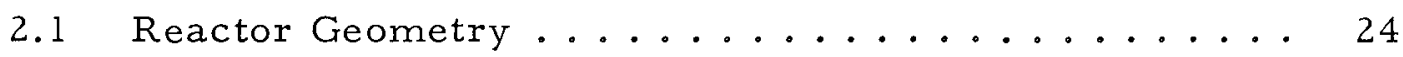

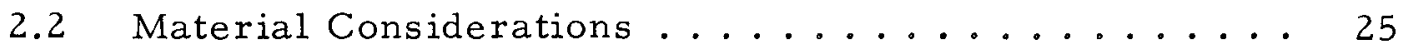

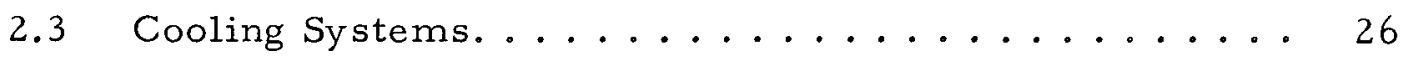

2.4 Physical Characteristics of the Coolant and the

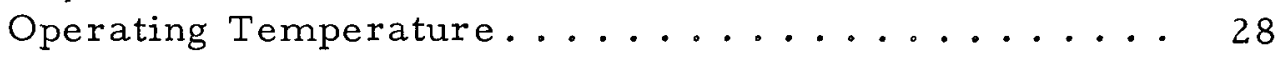

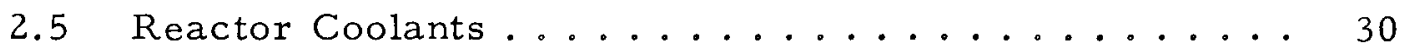

2.6 Varied Methods of Reactor Control .......... 31

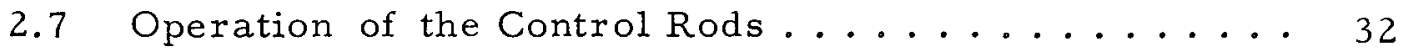

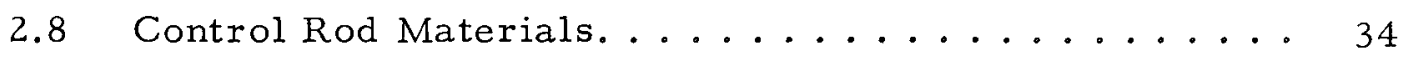

2.9 Control Rod Nomenclature and Requirements ...... 35

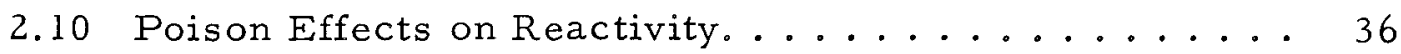




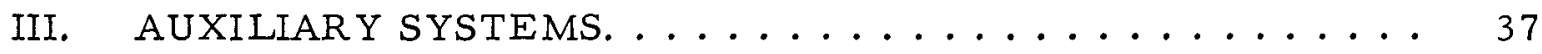

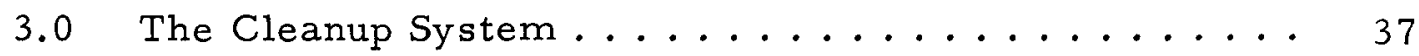

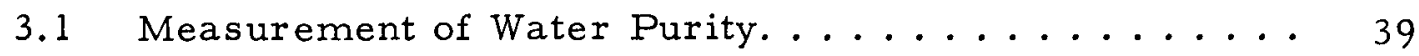

3.2 Conductivity Measurement.............. 40

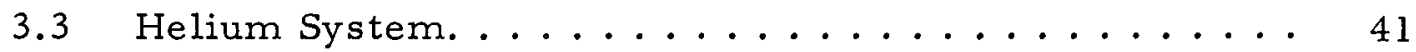

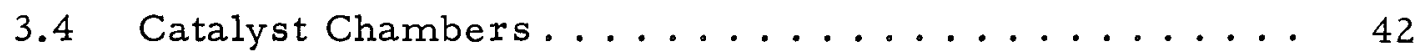

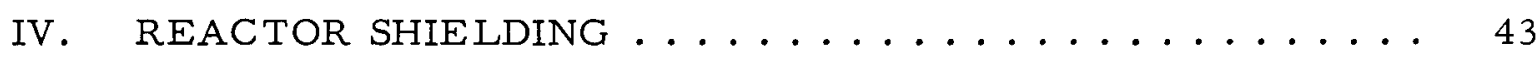

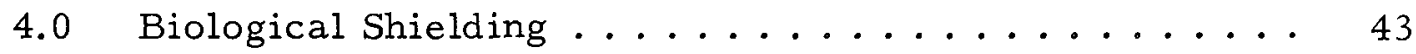

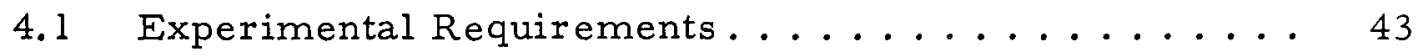

4.2 Radiations Produced in Fission. . . . . . . . . . 44

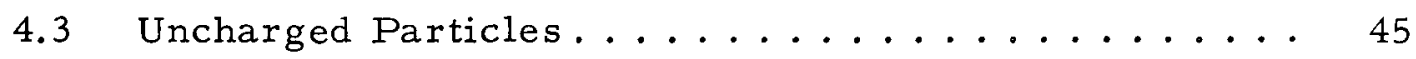

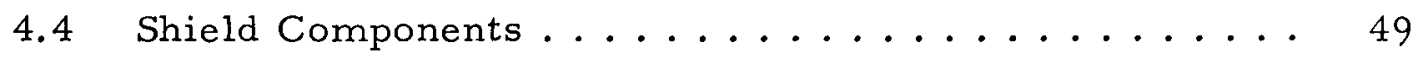

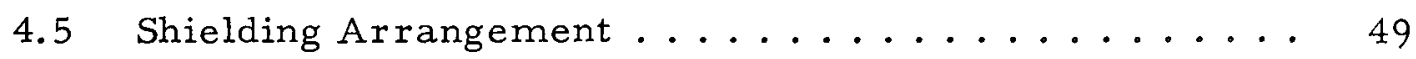

4.6 Irradiation Facility Shielding ............ 51

V. REACTOR TEMPERATURE AND PRESSURE

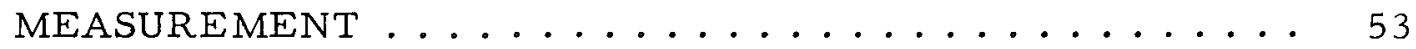

5.0 Necessity of Temperature Measurement........ 53

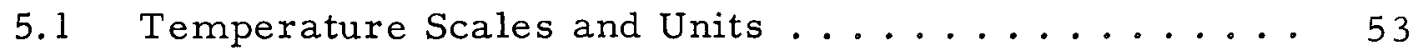

5.2 Temperature-measuring Devices .............. 55

5.3 Necessity of Pressure Measurement ......... 58

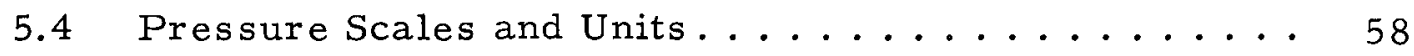

5.5 Pressure-measuring Devices................. 59

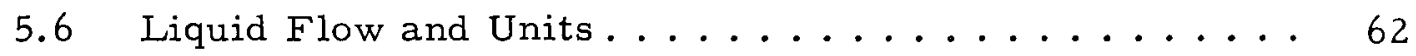

5.7 Liquid Flow-measuring Devices ............ 62

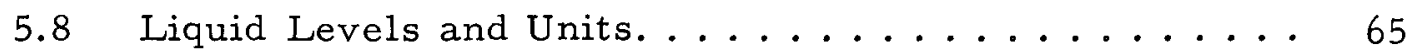

5.9 Devices for Measurement of Liquid Level. . . . . . 65 


\section{TABLE OF CONTENTS}

$\underline{\text { Page }}$

VI. NUCLEAR INSTRUMENTS. . . . . . . . . . . . . 69

6.0 Necessity of Nuclear Detectors and Ranges......... 69

6.1 The Ionization Chamber - Uncompensated and Compensated................. 70

6.2 The $\mathrm{BF}_{3}$ Proportional Counter ........... 73

6.3 Detector Circuits.................. 74

6.4 Flux Channels ...................... 75

6.5 The Period Channel ................... 76

6.6 Automatic Control Circuits - Regulating Rod ...... 77

6.7 Reliability of Safety Systems ............ 78

GLOSSARY ............................. 81 


\section{LIST OF FIGURES}

No.

Title

Page

1.0

Moderation of Neutrons by Elastic Collisions .......

1.1

Slow-neutron Fission Yield Curve for $\mathrm{U}^{235}$

1.2

Typical Determination of Critical Mass of $\mathrm{U}^{235}$

Effect of Delayed Neutrons on Power Increase.......

Positron and Electron Interaction.......... 48

5.0 Comparison of Fahrenheit and Centigrade Temperature Scales ......................... 54

5.4A\&B Diaphragm Pressure Gage............... 60

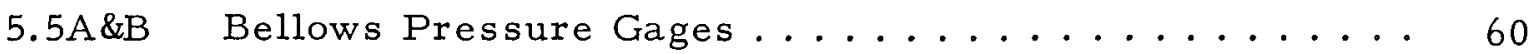

Differential Pressure Measurement - Diaphragm. . . . . 


\section{LIST OF FIGURES}

No.

Title

Page

5.13 Liquid-level Measurement by Sight Glass........ 66

5.14 Liquid-level Measurement by Float........... 66

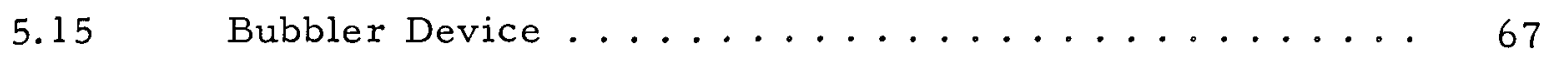

$6.0 \quad$ Multiple Instrument System ................... 70

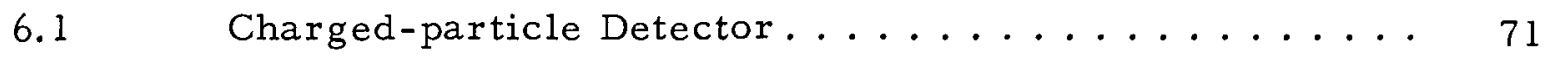

6.2 Uncompensated Ionization Chamber............... 72

6.3A\&B Gamma-compensated Ionization Chamber........ 72

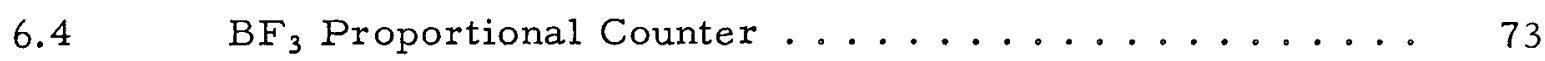

$6.5 \quad$ Neutron and Gamma Pulses ................ 74

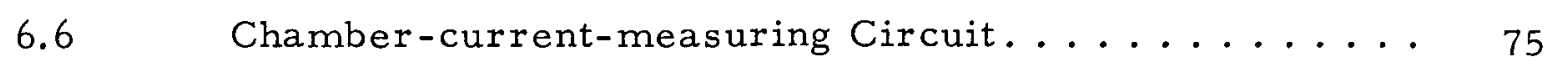

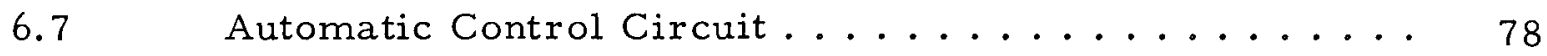

TABLE

No. Title Page

3.0 Relation of $\mathrm{pH}$ Value to $\mathrm{H}^{+}$and $\mathrm{OH}^{-}$Concentrations .... 39 


\title{
BASIC NUCLEAR AND REACTOR INFORMATION FOR THE REACTOR OPERATOR
}

\author{
Edited by \\ E. G. Greenman
}

\section{INTRODUCTION}

The typical research reactor exists to provide a source of radiation for scientific purposes and serves as a valuable proving ground for advancing reactor technology. The primary functions of the reactor operator must be the safe, efficient operation and maintenance of the various reactors and their components. An operator must be proficient in the normal routine operation of the reactor and also exhibit an active interest in reactor safety, economy, and efficient operation.

Operating a reactor competently requires complete understanding of all piping and valves for every system supplying the reactor. Nuclear instruments also require much study. The operator must understand the basic principles of the varied types of measuring devices and be able to locate all points at which measurements are made. Conditions often develop during normal operation that require immediate operator attention, and only complete knowledge of the reactor will enable the operator to cope with these situations. Individual proficiency and alertness will prevent needless loss of research time due to unscheduled reactor shutdowns.

All existing emergency procedures should be studied until they become automatic. As a member of a reactor team, safety of both reactor and personnel may depend upon the ability of the operator to do his job should an emergency condition ever arise.

Health Physics personnel are assigned to each reactor. Standard operating procedures have been established for the insertion and removal of experimental samples and for the handling of contaminated materials. An understanding of the functions and problems of the health physicist will enable the operator to do a better job.

It is hoped that this book will answer some of the questions that new personnel may have and provide impetus for further study. Every facet of reactor design, construction, and operation exists for a specific purpose. The understanding and application of these governing principles will lead to the development of good reactor operators, and enable them to cope with some of the many problems encountered in the reactor field. 


\section{CHAPTER I}

\section{THE FISSION PROCESS AND THE CHAIN REACTION}

\subsection{Spontaneous and Induced Fission}

Within the confines of a reactor vessel will be found an active core in which fission occurs and in which a controlled fission chain reaction may be sustained. The term fission, literally speaking, means to split or break apart. Biologists originally spoke of fission in reference to the splitting or regeneration of cellular structures. In nuclear fission, the process involves a splitting of the nuclei of various isotopes, with an attendant energy release. When a neutron is absorbed by a uranium or other fuel nucleus, there is a finite probability that this nucleus will split into two or more fragments. This process is restricted to heavy nuclei. In general, a nucleus will be too stable to suffer fission unless its isotopic mass is greater than the total mass of the particles into which it might be subdivided.

Fission may be spontaneous or it may be triggered by a subatomic particle, such as the neutron. The examination of one gram of natural uranium shows that approximately 25 nuclei undergo spontaneous fission every hour without the influence of any outside source. In neutron-induced fission, however, a nucleus of the appropriate type captures or absorbs a neutron. The resultant highly excited and unstable nucleus then splits into two or more pieces called fission fragments.

\subsection{The Moderation Process}

The neutrons in a nuclear reactor are not all of the same energy, but are distributed over a velocity range that extends from very slow to very fast neutrons. Fast neutrons are continually being produced by the nuclear fissions occurring within the reactor and slow neutrons are steadily being removed by absorption processes that lead to further fissioning and the creation of new fast neutrons. To compensate for the steady loss of slow neutrons through absorption and other losses, fast neutrons must be slowed down rapidly and efficiently to replenish the supply of slow neutrons. This process is called "moderation" or "thermalization."

The rapid transformation of fast neutrons to slow neutrons is accomplished by a moderation material or moderator that is incorporated in the reactor. The presence of a suitable moderator effects a slowing down of the fast fission neutrons by elastic collisions between the moderator nuclei and the neutrons until the average kinetic energy of the neutrons is somewhere near the energy of the moderator molecules (see Figure 1.0).

When a thermal equilibrium has been established between neutrons and the moderating material, the neutrons are said to be thermalized or 


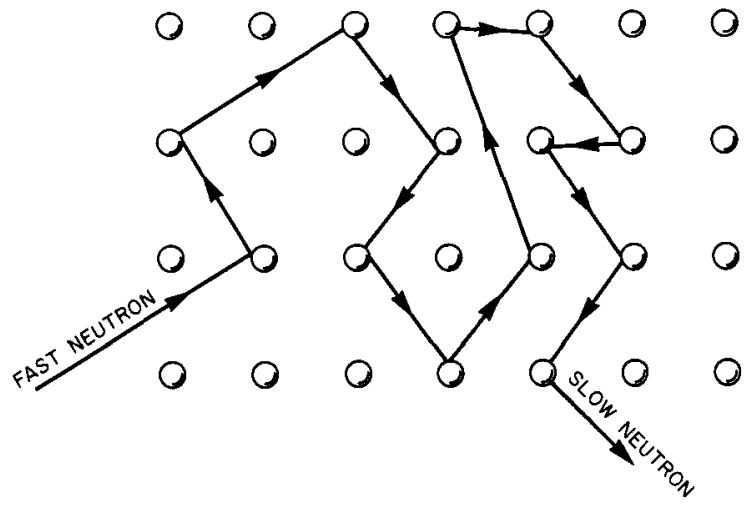

Fig. 1.0. Moderation of Neutrons by Elastic Collisions are called thermal neutrons. It is the slow or thermal neutron that is readily absorbed by the $\mathrm{U}^{235}$ nucleus in undergoing fission.

The choice of a suitable moderating material is determined by the consideration that its scattering cross section should be much larger than its absorption cross section in order to allow the colliding neutrons to reach thermal energies rapidly and to decrease the chance of their being absorbed. The cross section for any reaction is a measurement of the probability of the occurrence of that reaction under certain prescribed conditions. Thus, we speak of a scattering cross section when referring to a nuclear scattering process, an absorption cross section when dealing with absorption processes, and a fission cross section when considering nuclear collisions that lead to the fission of a nucleus.

\subsection{Fissionable Isotopes}

The two nuclides to be considered in this discussion are uranium235 and plutonium-239. These isotopes are abbreviated as ${ }_{92} \mathrm{U}^{235}$ and ${ }_{94} \mathrm{Pu}^{239}$, respectively. The letter symbols represent the names of the isotopes. $U$ is the chemical symbol for uranium and $P u$ the symbol for plutonium. The subscript in the symbol is called the atomic number and represents the total number of positive charges within the nucleus of one particular atom of the species. It is these positive charges or protons that determine many of the chemical characteristics of any element. The superscript is called the atomic weight or atomic mass and signifies the total number of particles, i.e., the number of protons and neutrons within the nucleus. From the foregoing description it may be seen that each atom of uranium-235 contains 92 protons, and a total of 235 protons and neutrons; also, that each plutonium-239 atom contains 94 protons and a total of 239 protons and neutrons.

Consider ${ }_{92} \mathrm{U}^{234},{ }_{92} \mathrm{U}^{235}$, and ${ }_{92} \mathrm{U}^{238}$, all isotopes of uranium. It is apparent that these three isotopes all have the same atomic number and differ only in the total number of particles within the nucleus. Isotopes then, are species of elements having the same atomic number, and hence the same chemical properties, but different atomic weights. Hence, all isotopes with atomic number 92 are called uranium. This principle holds true in the naming of all isotopic forms of any element. 
Natural uranium is composed of 0.0055 per cent uranium-234, 99.27 per cent uranium-238, and 0.72 per cent uranium-235. Uranium-235 is readily fissionable when it captures slow neutrons. This isotope comprises much of the nuclear fuel in reactors such as CP-5, Janus, and Juggernaut. The fuel used in these reactors contains approximately 93 per cent uranium-235 and is said to be "fully enriched." Although the process of obtaining a high concentration of the isotope is involved and relatively expensive due to the small percentage present in natural uranium, concentration of this isotope becomes necessary, as the other uranium isotopes are not fissionable by the capture of slow neutrons.

In a core with uranium-235 as fuel, reactions of the following type occur:

$$
{ }_{92} \mathrm{U}^{235}+{ }_{0} \mathrm{n}^{1} \rightarrow\left({ }_{92} \mathrm{U}^{236}\right) \rightarrow \mathrm{x}+\mathrm{y},
$$

where ${ }_{0} \mathrm{n}^{1}$ is a slow neutron, and $\mathrm{x}$ and $\mathrm{y}$ are primary fission fragments. The uranium-236 isotope, in most cases, exists only momentarily prior to the formation of the fission fragments $x$ and $y$.

\subsection{Production of Plutonium}

Plutonium-239 does not occur naturally, but must be artificially made. This isotope is fissionable by slow neutrons, and although at present there are few operating plutonium reactors, the isotope will very likely be more commonly used as fuel in the near future. Plutonium-239 is currently important in reactors using fuel rich in uranium-238. The plutonium product, capturing neutrons, causes reactor characteristics to change with time.

Examine the action of a fast neutron on uranium-238 (see Equation l.b below). The nucleus captures or absorbs the neutron and is transformed into uranium-239 accompanied by the emission of a gamma $r a y$. One neutron in the newly formed uranium-239 will change spontaneously into a proton, and a beta particle will be emitted. Half of all uranium-239 present at a given time will be converted into neptunium-239 in $23.5 \mathrm{~min}$ (see Equation l.c below). The time that it takes for one-half of the originally present nuclei to transform themselves is called the half-life. One neutron in the neptunium isotope changes into a proton, and the nucleus is transformed into plutonium-239 with the emission of a beta particle (see Equation 1.d below). The half-life of neptunium-239 is 2.33 days.

$$
\begin{aligned}
& { }_{92} \mathrm{U}^{238}+{ }_{0} \mathrm{n}^{1} \rightarrow{ }_{92} \mathrm{U}^{239}+\gamma(\text { gamma }) \\
& { }_{92} \mathrm{U}^{239} \stackrel{\text { Decays }}{\longrightarrow}{ }_{93} \mathrm{~Np}^{239}+\beta \text { (beta) } \\
& { }_{93} \mathrm{~Np}^{239} \stackrel{\text { Decays }}{\longrightarrow}{ }_{94} \mathrm{Pu}^{239}+\beta \text { (beta) }
\end{aligned}
$$


The half-life is a measure of the time required for one-half of a given sample to be transformed by decay. It may be defined as the time required for the radioactivity of a given amount of an element to decay to one-half its initial value. If we had started at a particular time, with $16 \mathrm{gm}$ of neptunium-239, at the end of 2.33 days we would have $8 \mathrm{gm}$ of the original element, and $8 \mathrm{gm}$ of plutonium-239. The initial neptunium nuclei each had 93 protons and 146 neutrons. (The atomic weight minus the atomic number equals the number of neutrons.) The beta-decaying nuclei formed a product with 94 protons but only 145 neutrons. The missing neutron changed to a proton, an electron, and a neutrino.

At this point, we might briefly consider the possibility of using plutonium as a means of creating additional fissionable material within a reactor. Let us examine a reactor whose fuel consists of one per cent uranium-235 and 99 per cent uranium-238. This combination of fuel is sometimes referred to as "slightly enriched." Plutonium isotopes are generated as follows: The $U^{235}$ nuclei undergo fission, liberating free neutrons in the process. Some of these neutrons are captured by the $U^{238}$ nuclei, and through the intermediate processes create $\mathrm{Pu}^{239}$, which, as previously stated, is a fissionable isotope. During early stages of irradiation, neutrons captured by $\mathrm{U}^{238}$ to create $\mathrm{Pu}^{239}$ come almost entirely from $\mathrm{U}^{235}$ fission. After a time, the $\mathrm{U}^{235}$ burns up and $\mathrm{Pu}{ }^{239}$ builds up, so more and more of the total number of neutrons within the reactor will be generated from fission of the $\mathrm{Pu}^{239}$. A maximum point is reached in $\mathrm{Pu}^{239}$ concentration when the plutonium is being formed at a rate equal to that at which it is being used as fuel.

\subsection{Fission Yield of Uranium-235}

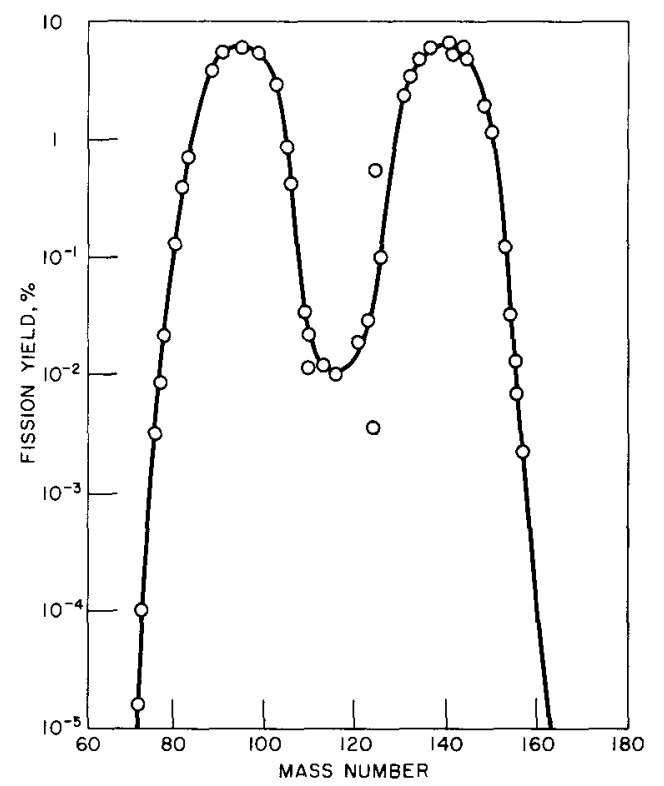

Fig. 1.1. Slow-neutron Fission Yield Curve for $\mathrm{U}^{235}$
Uranium-235 will undergo fission when bombarded by slow or thermal neutrons; neutrons of higher energies will also produce fission. It is significant that the nucleus does not split into two equal halves and also that it does not always fission into the same fragments. The uranium-235 nuclei are capable of forming more than 40 different isotopes. A great deal of work has been done towards the identification of these various products, and a curve of the fission yield (see Figure 1.1) has been established.

The fission yield is defined as the percentage of nuclear fissions yielding products of a given mass number. The mass number rather than the atomic 
number is plotted, as these fragments, or fission products, are highly radioactive and subject to decay. This means that the atomic numbers of these products will change with time, whereas the mass numbers for the most part remain unaffected, such as the formation of uranium-239 into neptunium-239 as described by Equation (1.c). The atomic weight or mass number remains constant, but the atomic number increased from 92 to 93.

When a curve of the type shown in Figure 1.1 is prepared, it states basically that, for a given number of fissions, a certain probability exists for fission products of a particular mass number to be formed. In examining this curve, it is apparent that the fission products may be divided into two main groups. The first is a "light" group with mass numbers ranging from 85 to 104 , and the second is a "heavy" group with mass numbers of 130 to 149. If we were experimentally to sample all of the fission products contained in a reactor using enriched uranium-235 as fuel, a distribution following this fission yield curve would result.

\subsection{Important Fission Products}

Certain of the fission products formed within reactors have a definite effect on reactor characteristics, whereas others, due to various properties, serve as guides in providing operating personnel with a tangible picture of events taking place within the reactor vessel and core regions.

Consider xenon-135. This isotope is formed primarily from the beta decay of tellurium-135. As a result of this decay process, which takes place with a definite half-life, xenon-135 continues to form in considerable amounts, even after a reactor is shutdown. The isotope has a very high cross section for neutron absorption and if a sufficient concentration is accumulated will terminate the chain reaction. This isotope acts as a parasite, absorbing neutrons which would otherwise be used to sustain the fission chain.

A reactor must not be shut down during normal running periods for too long a time or the presence of xenon-135 may poison the chain reaction. If this happens, it will be necessary to wait some hours for the xenon-135 to decay to a sufficient extent to permit startup. The peak in xenon-135 concentration occurs approximately $11 \mathrm{hr}$ after shutdown. As the amount of xenon-135 formed depends on the neutron intensity, this isotope causes considerable problems in reactors with very high neutron fluxes. Ultimately, the radioactive decay of the xenon-135 to cesium-135 will reduce the total concentration of xenon-135 to such a level that the reactor may be operated.

Once a reactor is operating and stable at a given power level, and the rate of production of the xenon-135 isotope is equal to the rate of transformation into xenon-136 by slow-neutron capture, xenon-135 concentration will remain constant and the reactor can continue to operate. 
The fission products found within a reactor are tremendously radioactive. In at least one stage of their development, almost all of the fragments exist in a gaseous phase, which enhances the possibility of escape. For this reason, fuel sections are carefully clad and rigidly inspected. The cladding keeps these radioactive products inside the fuel element and prevents radioactive contamination of reactor systems.

Cesium-137 is a fission product that emits a characteristic gamma ray which is easily detectable. Reactor systems, particularly coolant lines, are often monitored for the presence of this isotope to detect the presence of a leak in the fuel clad.

\subsection{Energy Released from Fission}

In the fission of uranium, the sum of the masses of the fissioning uranium nucleus plus a neutron is greater than the sum of the masses of the fission products, $x$ and $y$. The difference in mass has been converted into energy. The amount of energy released may be calculated through use of equation $E=M C^{2}$, in which $E$ is the energy in ergs, $M$ is the mass lost in grams, and $C$ is the speed of light, $2.99 \times 10^{10} \mathrm{~cm} / \mathrm{sec}$. It has been calculated that for each individual fission a mass of $3 \times 10^{-25} \mathrm{gm}$ is lost. In considering this decrease, it should be apparent that only a very minute amount of matter is actually converted into energy. If it were possible to convert one gram of mass instantaneously into energy, the energy released would be tremendous.

The energy released in fission appears in part as kinetic energy or the energy of motion of the fission fragments. Fission products are charged and relatively heavy. They stop quickly in the metal of the fuel elements, and almost all of their kinetic energy is given up as heat energy. It requires $3.1 \times 10^{10}$ fissions per second to produce energy at the rate of one watt.

Fuel burnup is not to be confused with mass decrease. A reactor operating at one megawatt uses approximately one gram of uranium-235 per day. This means that $2.56 \times 10^{21}$ atoms have fissioned, but since only $3 \times 10^{-25} \mathrm{gm}$ is converted into energy per fission, the reactor actually converts $0.0008 \mathrm{gm}$ into energy per megawatt day. The rest of the uranium used is converted into fission fragments, not energy, and hence is lost as far as being able to help sustain the reaction is concerned. Once uranium235 has undergone fission, most of the original mass is contained in the fission products.

\subsection{The Chain Reaction}

As a uranium-235 nucleus fissions, it emits neutrons in addition to the various fission products formed. The same number of neutrons is not always released from each fission. On the average, 2.47 neutrons will be 
liberated per fission. A fraction of a neutron is never released. Sometimes two and at other times three neutrons will be liberated in the fission process. Only one neutron is required to cause a uranium nucleus to fission, and, since each fission provides 2.47 neutrons on the average, it is seen that, under the correct conditions, the original fission might cause further fissions. In a chain reaction, each nucleus must capture one neutron, undergo fission, and produce at least one neutron which causes the fission of another nucleus. When this occurs, a chain reaction will be sustained. The intensity of the chain reaction process will increase unless controls are applied so that of the 2.47 neutrons liberated per fission only one will give rise to an additional fission.

\subsection{The Neutron Balance}

In theory, achieving a critical condition would seem to be relatively simple, but in practical application this is not the case. A reactor must be of finite size, and as a result some of the initial number of neutrons available for fissioning escape from the reactor.

In addition to the problem of reactor size, the heat energy arising from the fission fragments must be removed by coolants. Installation of coolant and other systems allows additional neutrons to escape through, or be absorbed by, structural materials of these components. A certain percentage of neutrons is absorbed by nuclei that do not undergo fission and this absorption results in a further loss of available neutrons. All of these factors must be taken into consideration in the development of a condition where the neutrons produced, minus the neutrons escaping, minus nonfissionproducing neutron absorption, is equal to one. This means that after all these events occur, one neutron must still be left that will produce an additional fission.

\subsection{Subcritical Multiplication}

A reactor has three distinct and separate conditions: subcritical, critical, and supercritical. When a reactor is subcritical, the neutron balance is less than one, i.e., less than one neutron per fission can cause another fission, and the reaction is not self-sustaining. Consider a subcritical reactor having either an external or internal source of neutrons. This stream of neutrons encounters uranium-235 nuclei and some fission occurs. These fissions yield a multiplication of the number of neutrons from one generation to the next.

In a reactor, if the neutron-balance equation yields a number that is less than one, such as 0.9 , from generation to generation, the number of neutrons available to produce the next generation will be less than those which made the preceding generation; the reactor is then subcritical. If the neutron-balance equation yields a number greater than one, the reactor is supercritical, and if the number is just equal to one, the reactor is critical. 
When a reactor is stable at any power level, regardless of what the power level might be, the neutron-balance number must be equal to one. This number is called the multiplication factor or $k$, and basically means the multiplication of neutrons from one generation to the next. Reactors are controlled by modifying the value of $k$. To attain a subcritical condition, movable neutron absorbers are inserted into the reactor core to decrease multiplication, making $k$ less than one. To reach criticality, some of these absorbers are removed from their proximity to the core, until $\mathrm{k}$ is equal to one. To operate at a certain power level more absorbers are removed until $k$ becomes greater than one. When this happens, the number of fissions increases, and the power rises. Once the desired level is reached, $k$ is again reduced to one, and the reactor may be maintained at a stable critical level for as long as may be desired. It might be critical, at $10 \mathrm{kw}$ or $10 \mathrm{Mw}$, but $\mathrm{k}$ would still equal one, in either case.

\subsection{Approach to Critical}

The multiplication factor $k$ may be altered by the addition or subtraction of fuel. If fuel is added, more nuclei are present to undergo fis sion, and $\mathrm{k}$ will be increased. When a reactor is loaded with fuel, the fissionable material is carefully introduced in small quantities while measurements are made to determine the value of $\mathrm{k}$.

Consider a source, either external or internal, yielding $s$ neutrons per second. If the reactor is subcritical, $k$, by definition, is less than one. Let $\mathrm{N}$ be the number of neutrons in the core. Before any generation occurs, the reactor contains $s$ neutrons. These neutrons encounter fuel and are increased by the multiplication factor $k$. We now have $s+k s$ neutrons after the first generation of fissions. These newborn ks neutrons also encounter fuel and are increased by $k$ to form $k^{2}$ s neutrons. This process continues through all generations and may be expressed as follows:

$$
\begin{aligned}
& \mathrm{N}_{1}=\mathrm{ks} \\
& \mathrm{N}_{2}=\mathrm{s}+\mathrm{ks} \\
& \mathrm{N}_{3}=\mathrm{s}+\mathrm{ks}+\mathrm{k}^{2} \mathrm{~s} \\
& \mathrm{~N}=\mathrm{s}+\mathrm{ks}+\mathrm{k}^{2} \mathrm{~s}+\ldots \mathrm{kj}_{\mathrm{s}}
\end{aligned}
$$

The relationship between neutrons, arising from both source and fission, and the multiplication factor may be expressed in terms of the subcritical multiplication equation. This equation is derived as follows:

$$
N=s\left(1+k+k^{2} \ldots k^{j}\right) \quad \text {. }
$$

Now, 


$$
\left(1+k+k^{2}+\ldots k j\right)=\frac{1}{1-k} \quad, \quad \text { if } k<1 .
$$

Accordingly,

$$
\mathrm{N}=\frac{\mathrm{s}}{\mathrm{l}-\mathrm{k}} \text {. }
$$

In adding fuel to a reactor, we must locate the point at which the reaction ceases to be subcritical, and becomes just critical. The subcritical multiplication equation may be written in the form

$$
\frac{\mathrm{s}}{\mathrm{N}}=1-\mathrm{k}
$$

When a reactor is critical, $k$ is equal to one; therefore,

$$
\frac{s}{N}=1-1=0 .
$$

This means that $N$, or the number of neutrons after $j$ generations, must be extremely large and approaching infinity, if $\mathrm{s} / \mathrm{N}$ is to be equal to zero. Advantage may be taken of this fact to construct a revealing plot.

When no uranium is in a reactor, the only neutrons present are source neutrons. These neutrons are counted by electronic instruments

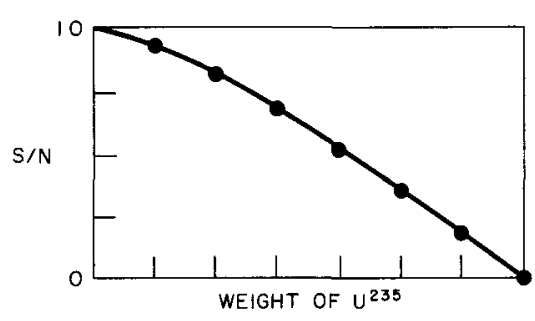

Fig. 1.2

Typical Determination of Critical Mass of $U^{235}$ prior to the initial fuel loading. As fuel is added, $\mathrm{N}$ is increased due to multiplication. Further neutron counts are made with each fuel addition, and a curve such as illustrated in Figure 1.2 is plotted from these points. After several points have been established, it is possible to approximate the point where enough uranium is present to make $s / N=0$. This point is the total amount of fuel required for a critical mass.

A curve of this nature is very important in setting a limit to the amount of fuel loaded into a reactor. Particular attention must also be directed to the role of the source neutrons in this operation. These neutrons must be of sufficient intensity to be monitored and counted by reactor instruments. If the source neutrons are too few to yield a valid factor for the plot, it might be possible to add too much fuel.

A reactor such as CP-5 contains a built-in source within the spent fuel elements. A spent element containing fission products is left in the 
reactor and used for startup after fuel changes. The fission products within the fuel element emit gamma rays of high intensity and energy which displace neutrons from the deuterons in the molecules of the heavy water. These displaced neutrons are then available for fission.

In a light-water reactor, neutrons may be liberated from an artificially activated source such as antimony and beryllium. The activated antimony emits gamma rays of sufficient energy to displace some of the neutrons from the beryllium, which are then available for fission.

\subsection{Supercritical Behavior}

When a reactor is in a supercritical condition, $k$ is greater than one, and the reactor power increases. The amount that $k$ exceeds one is described as $k$ excess or excess reactivity. As $k$ excess is usually a very small number, and never very far from one, it is expressed in per cent. When $k$ is equal to one, $k$ excess equals zero. Thus, $k$ excess may be expressed as

$$
k_{\text {ex }}=k-1
$$

Consider a simplified version of a reactor increasing in power. The equation describing the increase in power is

$$
P_{f}=P_{i} e^{\frac{k_{e x} t}{\ell}}
$$

where

$$
\begin{aligned}
P_{f} & =\text { final reactor power } \\
P_{i} & =\text { initial reactor power at any level, } \\
e & =2.718 \\
k_{e x} & =k-1 \\
t & =\text { time, } \\
\ell & =\text { neutron lifetime or time between generations. }
\end{aligned}
$$

The quantity $k_{e x} / l$ is usually replaced by $1 / \tau$. The symbol $\tau$ is the letter "tau" of the Greek alphabet. If this symbol is used, the equation may be rewritten in the form

$$
P_{f}=P_{i} e^{t / \tau}
$$

Assume that the reaction continues until $t=\tau$; then,

$$
P_{f}=2.718 \mathrm{P}_{i}
$$


This means that the power at a given time $t$, will have been increased by a factor of 2.718 over the initial starting power. The time $t$ is equal to $\tau$ and is called the period. It is defined as the time required for a reactor to increase in power by a factor of e, or 2.718 .

The time required for a reactor to double its power level may also be calculated when the period is known:

$$
\text { Doubling time }=(0.693)(\text { period }) .
$$

If only the doubling time is known, the reactor period may be calculated from a different form of the same equation:

$$
\text { Period }=\text { Doubling time } / 0.693 .
$$

\subsection{Effect of Delayed Neutrons}

In writing a simplified power formula such as Equation 1.1, it was assumed that all of the fission neutrons were born instantaneously (that is, were prompt neutrons). This would mean that the neutron density and energy output would increase with extreme speed and that automatic mechanical control devices might not be sufficiently rapid in halting a power rise. Fortunately, a small percentage of neutrons are not released immediately in the fission process, but are born after a slight time delay. These delayed neutrons, as they are called, are released from decaying fission products. In the case of uranium-235 fission, the delayed neutrons constitute a fraction 0.0068 of the total neutron population.

The power of a hypothetical reactor with prompt neutrons alone, supercritical by a given amount, would increase approximately 8,000 times

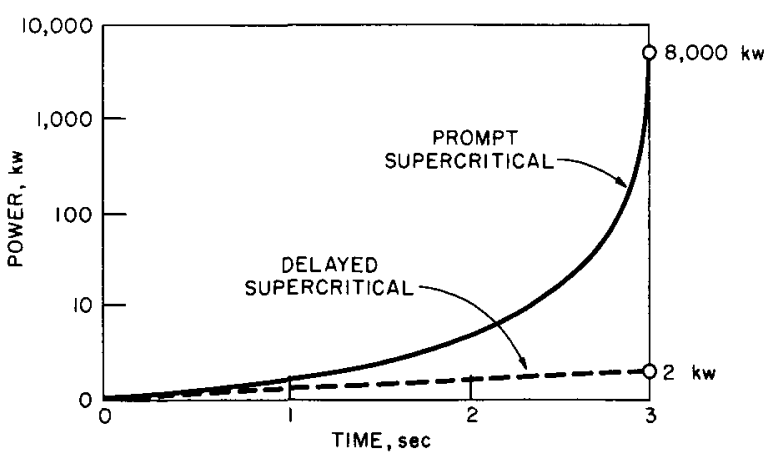

Fig. 1.3. Effect of Delayed Neutrons on Power Increase in an elapsed time of $3 \mathrm{sec}$. An actual reactor, dependent upon the delayed-neutron fraction, only increases in power by a factor of two during the same period when supercritical by the same amount (see Figure 1.3). A relatively slow and gradual rise in reactor power will occur as long as the amount of excess reactivity does not exceed the delayed-neutron fraction. When the value of excess $k$ is less than 0.0068 , each succeeding generation of neutrons must wait for the preceding generation of delayed neutrons to be born, before it may be completed, and the reactor is "delayed critical." Of course, when $k$ excess exceeds the delayed-neutron fraction, each succeeding generation of fissions no longer needs delayed neutrons to be 
completed and the power rises rapidly. When a reactor becomes critical on prompt neutrons alone, it is said to be "prompt critical."

Another contribution to the delayed-neutron fraction comes from an interaction of gamma rays from the fission products with such materials as heavy water and beryllium, causing neutrons to be liberated. These gammainduced neutrons are called photoneutrons; as they also are delayed, these contribute to the delayed-neutron fraction. In CP-5, a heavy-watermoderated reactor, the delayed-neutron fraction is approximately 0.0076 as compared with a delayed-neutron fraction of 0.0068 found in light-water and graphite-moderated reactors. With increase in size of the delayedneutron fraction, each generation of neutrons must wait a little longer for the completion of the preceding generation. A beryllium-or heavy-watermoderated reactor is slower in response to reactivity changes than is a light-water- or graphite-moderated reactor.

\subsection{Temperature Coefficients}

Temperature plays an important role in the ability of a reactor to sustain a chain reaction. The effect of temperature enters through the socalled "temperature coefficient." As a chain reaction proceeds, heat energy is produced. This heat energy causes expansion of reactor components, resulting in an actual increase of reactor size. As this occurs, fewer neutrons escape from the system and additional neutrons are available for return to the core region. The additional neutrons, not lost through leakage, cause an increase in reactivity.

The moderating medium becomes less dense as its temperature increases and fewer scattering centers are available per unit volume; consequently, the velocity of the moderated neutrons increases, as neutrons are not reduced in energy as efficiently at higher temperature ranges. Furthermore, a moving moderator molecule cannot make a neutron which collides with it go any slower than the molecule is moving. Since the speed of the moderator molecules increases with temperature, neutrons moderated by a hot moderator are faster than those moderated by a cold moderator. Fast neutrons do not cause fission as well as slow neutrons, and so the reactivity decreases. The density change within the moderator also has a positive effect upon reactivity. As the density decreases, the number of neutrons absorbed decreases, and more neutrons become available for fissioning, thus increasing reactivity.

The various effects of temperature changes within a reactor should be balanced to insure that the net effect of a large temperature rise will be a reactor shutdown. The negative temperature coefficient is the factor which, when multiplied by the temperature, yields a decrease in reactivity. In the event of a nuclear incident, accompanied by a sudden temperature rise, a reactor with an inherent negative temperature coefficient will tend to shut itself down, thereby preventing damage to reactor components. 


\section{CHAPTER II}

\section{REACTOR STRUCTURAL COMPONENTS}

To understand the problems involved in selecting and working with various reactor components, a general reactor scheme will be discussed. Reactors are usually closed systems, and the center or heart of the system is the reactor core. The fuel, located in the core, may consist of uranium, thorium, or plutonium. The combination of these elements will depend upon the specific design of the reactor; any or all may be present.

As previously stated, this discussion is limited to the consideration of uranium-235 in a solid state. The fuel is generally in close proximity to or intermingled with moderating materials, such as light or heavy water, beryllium, carbon in the form of graphite, or other suitable light elements. This moderating material is necessary to slow down fission neutrons to the required energies. The combination of fuel, moderator, and associated structural and clad materials will be referred to as the core.

To remove heat from the reactor, a suitable heat transfer material is passed through the core. Gases, water, or liquid metals may be used, depending upon requirements of the system. Furthermore, in some reactors the moderator itself may be passed through the core as a heat transfer material.

A reflector is placed outside the core, which is used to conserve neutrons by "bouncing" them back into the core region. Surrounding the reflector is a biological shield which attenuates radiation coming from the core. This is usually a combination shield which attenuates both neutrons and gamma rays.

Control rods are located in the core or reflector regions and regulate the reactor power level by controlling the rate of production of neutrons. Basically, then, a nuclear reactor is a system consisting of a fuel containing fissionable material, a moderator, and heat-removal components in a geometric arrangement capable of sustaining a controlled chain reaction.

\subsection{Structural Form Versus Neutron Balance}

In choosing reactor structural forms, materials, and integral components, particular attention is directed to the neutron-balance equation. A sustained chain reaction depends upon the availability of a sufficient number of neutrons for the completion of each generation of fissions. The neutron, an electrically neutral particle, is not electrostatically repelled as it approaches various types of nuclei, as are charged particles. A free neutron is susceptible to capture or absorption in the formation of compound nuclei; therefore, in reactor construction, compromises must be made to insure a proper neutron balance. 
Consider a simplified representation of a nuclear reaction, in which 100 fast neutrons are liberated from fission (see Figure 2.0). The velocity of the 100 initial neutrons is de-

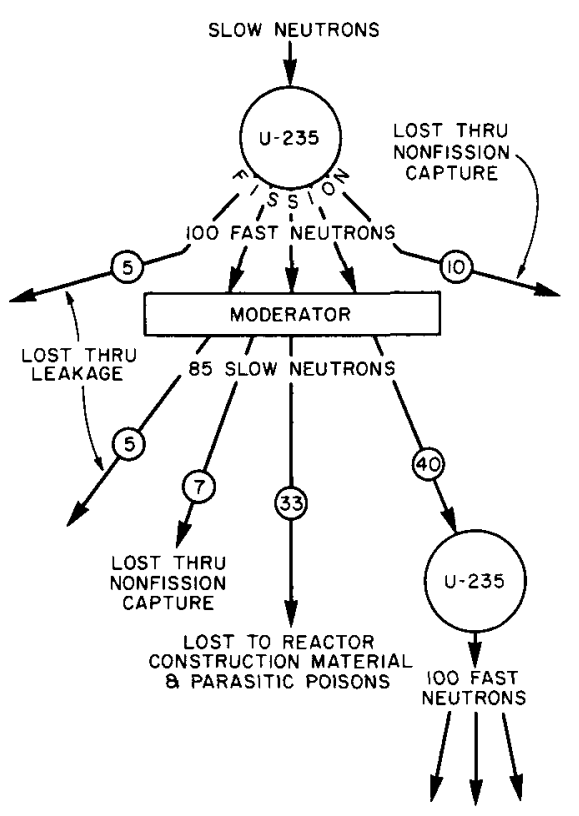

Fig. 2.0. Neutron Generation from $\mathrm{U}^{235}$ creased by a suitable medium to enhance fission capture. As this slowing down, or moderation occurs, 5 neutrons physically escape the reaction, or at least are lost to the finite zone of influence. An additional 10 neutrons will be captured by the fuel during the moderation process, forming nuclei that do not suffer fission. At this point, 85 slow or thermal neutrons are present, and, theoretically, each under proper conditions could cause a fission. However, 5 slow neutrons escape through leakage, 33 neutrons will be absorbed by parasitic reactor poisons, moderator atoms, and by various structural materials. Of the remaining 47 slow neutrons, 7 will be absorbed by the fuel without undergoing fission, and the other 40 neutrons will cause any fissions that occur. Each fission produces, on the average, about 2.5 free neutrons, and a fission reaction involving 40 neutrons would yield a secondary genera-

tion of 100 fast neutrons. This type of reaction will continue with time until fuel is expended or the neutron balance altered. Conditions may be altered by the use of materials that readily capture neutrons, or by the use of forms that enhance the probability of neutron leakage. Possibly under these and other conditions only 30 neutrons would actually cause fission. As each generation is completed, fewer and fewer neutrons would be liberated, and the reaction would decrease and become dependent upon source strength. If this situation occurred in a reactor, the reactor would shut down, and operation could not proceed until the neutron balance was altered. For this reason, considerable care is exercised in the choice and placement of various reactor construction materials. If an existing condition is altered, some other condition must be changed to have the opposite effect in order to maintain an equilibrium. Without neutron balancing a reactor cannot function.

\subsection{Reactor Geometry}

The fraction of neutrons escaping or leaking from a reactor may be reduced if the size of a reactor system is increased. The number of neutrons escaping depends upon the external surface area while the number of fission neutrons liberated is dependent upon the volume. Geometric considerations are therefore concerned with providing the greatest volume with the smallest surface area. In examining a cube with dimensions of 
$1 \mathrm{~cm}$, the volume is equal to $1 \mathrm{~cm}^{3}$ and the surface area equals $6 \mathrm{~cm}^{2}$. In this instance, the ratio of volume to area is $1: 6$. When each dimension of the cube is increased to $2 \mathrm{~cm}$, the volume equals $8 \mathrm{~cm}^{3}$ and surface area equals $24 \mathrm{~cm}^{2}$. A comparison of volume versus surface area yields a ratio of 8:24 or 1:3. As neutrons escape only through a surface area, it is readily apparent that decreasing the ratio of volume to area results in less neutron leakage.

The ideal geometric figure used in decreasing the ratio of volume to area is the sphere. More volume can be used with less neutron leakage within a sphere than for any other shape. The primary disadvantage of employing a spherical core lies in the difficulties encountered in construction. From an engineering standpoint, a sphere is costly and difficult to construct. In reactors using various types of liquids for heat removal, the spherical form of necessity must be disrupted by the insertion of various piping lines comprising the cooling systems. Due to the engineering difficulties encountered with spherical shapes, a core is usually shaped geometrically in the form of a right circular cylinder or as a cube. The cylinder has been of particular importance, and it is the type of core that is employed in reactors such as CP-5, Janus, and Juggernaut. Although a cylinder and cube enhance the possibilities of neutron leakage to a greater extent than in the case of the sphere, these forms are more feasible from a practical construction standpoint. In consideration of reactor design, emphasis is placed on the various compromises necessitated in maintaining a neutron balance.

\subsection{Material Considerations}

In reactor design, the strength of a structure is determined by the specific needs of the individual reactor system. In each system, data involving anticipated temperatures and pressures undergo careful analysis prior to the selection of a particular construction material. A reactor engineer uses this information to determine stress requirements and then adds an adequate safety factor.

As the selection of materials is made, particular attention is directed to the cross section for neutron absorption. This cross section represents the efficiency with which an individual nucleus of a particular species will react with a neutron. The effective cross section for neutron absorption is not constant, but varies for different materials. A unit of cross-section measurement called the "barn" has been adopted. This unit is equal to $10^{-24} \mathrm{~cm}^{2}$. It should be apparent that by choosing reactor materials with low nuclear cross sections, the problem of maintaining sufficient numbers of neutrons to sustain a chain reaction is diminished. 
Structural materials may be classified in three groups:

1. elements with low cross sections (that is, less than one barn);

2. elements having moderate cross sections (that is, 1 to 10 barns);

3. elements with high cross sections (greater than 10 barns).

Materials such as beryllium, magnesium, zirconium, and aluminum have cross sections less than 1 barn, which makes them useful as reactor materials. Beryllium, with the lowest cross section and a low atomic mass, possesses excellent moderating qualities, but its high cost limits the use of this element as a reactor material. Magnesium, with a slightly higher cross section, is also too expensive for general use. The cross section of zirconium is low enough to warrant its use, but this element has only recently become available in quantities of sufficient purity. The cross section of aluminum for neutron absorption is of an acceptable value, and the element is readily available and reasonably inexpensive. For these reasons, a great deal of aluminum is used in construction of a reactor.

The primary disadvantage in the use of aluminum is its susceptibility to corrosion at high temperatures. If requirements of a reactor do not justify the use of aluminum, elements falling within the group of moderate nuclear cross sections: columbium, iron, molybdenum, copper, nickel, vanadium, titanium, and chromium, may be considered. Iron, copper, nickel, and chromium are components of the better grades of stainless steel. In dealing with high temperatures or greater strength requirements, stainless steel components are often used and a compromise made regarding the requirements of the system and the higher cross section for neutron absorption. Elements found in the third group, having cross sections exceeding 10 barns, make very poor materials for reactor construction because of their pronounced detrimental effect upon the neutron balance.

\subsection{Cooling Systems}

One of the important items in the design of any reactor, is the reactor cooling system. A method of heat removal is necessary to prevent undesirable temperatures in reactor fuel, structural materials, and in the coolant itself. Cooling systems are classified as either direct cycle or indirect cycle.

In using a single-pass system, the coolant is forced through the reactor once and then returned to a heat sink. This manner of cycling is advantageous primarily due to its simplicity. A system of this type would be especially useful at reactor sites located in remote areas or near 
bodies of water. Either air or water may be used, depending upon requirements of the reactor and its location. The primary disadvantages include a necessity for purification when water is used, and adequate holdup or disposal facilities to prevent discharge of contaminated water or air into the surrounding area. Lake or river water contains minerals and other impurities, some of which are subject to induced radioactivation and have corrosive effects on reactor components. At higher power levels, of course, air is not satisfactory as a coolant due to its chemical activity at high temperatures.

The indirect cycling method of heat removal is much more feasible for reactor use and provides considerably more flexibility both in location of a reactor and in the choice of acceptable coolants. This type of cooling uses a closed primary system and one or more successive separate coolant circuits. Most reactors are cooled by some application of this method. A closed circuit serves to contain many of the radioactive materials and eliminates the need for elaborate disposal facilities. The primary disadvantage in using such a system is one of cost.

In indirect cycling, the heat energy is transferred from the fuel elements to the primary coolant as it passes through the core region. The primary coolant functions to contain induced radioactivity and to transport the heat energy to the secondary system. The coolant usually passes through a heat exchanger, and the heat removed is transmitted to a heat sink or an additional system. The heat sink is simply a medium by which heat is dissipated.

Consider a simplified flow diagram, in which the primary coolant is a liquid (Figure 2.1). The liquid in the primary system is forced through the reactor vessel by a suitable pump in a closed loop. A heat exchanger comprises one portion of this system. A secondary system originating in the basin of a cooling tower containing ordinary water also enters one section of the heat exchanger. The heat energy from the reactor is transferred from the primary system to the secondary.

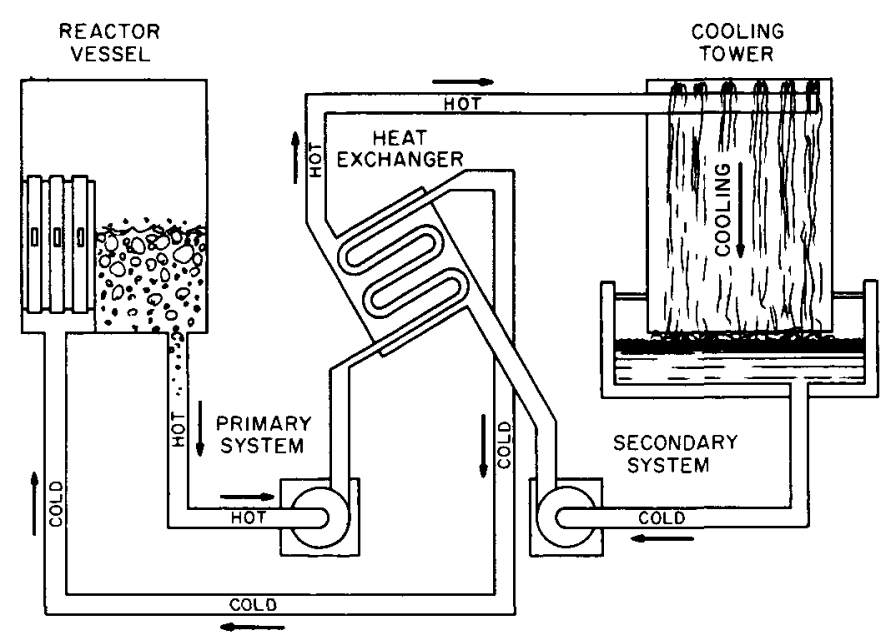

Fig. 2.1

Simplified Flow Diagram Cooling Systems 
The water in the secondary system is also forced through the heat exchanger and then returned to the tower. The heated water is sprayed through a lattice-type structure from the top of the tower, and heat is transferred to the atmosphere prior to re-entry of the water at the tower basin. In a power reactor the heat generated may be used either to operate turbines or may be sent through coils to produce steam, as requirements of the particular reactor may demand.

In addition to the primary and secondary systems of a reactor, further cooling is provided within the reactor reflector and shield. Approximately 5 per cent of the total heat generated within a reactor is absorbed within the reflector and shield. This heat is a direct result of the absorption of gamma radiation escaping from the core. As the gamma radiation is stopped, its energy is converted to heat. This heat must also be removed to prevent structural damage to the shield. Normally, copper coils are embedded throughout the shield and extending completely around it. These coils are filled with distilled water which serves to transfer the heat from the shield to another cooling system.

\subsection{Physical Characteristics of the Coolant and the Operating Temperature}

The operating temperature of any reactor depends to a considerable extent upon certain physical properties of the coolant and its flow rate through the core. Heat removal is a function of the flow rate, and an increase in the flow rate causes a rise in the amount of heat removed for a given time.

Heat removal is, of course, a combination of convection, conduction, and radiation. In referring to the coolant and its characteristics, we will consider only convection. Convection is simply the transference of heat by moving masses of matter, either gaseous or liquid. Convection may be natural as observed in gases, whose movement may be caused by differences in densities, or it may be forced by various pumps or fans. In early reactors, the heat produced was insignificant, and natural convection provided adequate cooling. As reactor technology advanced, reactors of higher power levels were constructed, and it became necessary to use forced convection to circulate the coolant. In most present-day reactors the heat produced is sufficient to warrant use of forced-convection methods. The properties of the coolant and the amount of heat will determine the pumping power required for adequate cooling.

The maximum operating temperature of heterogeneous reactors is determined by the maximum permissible temperatures of the solid reactor components and the fuel elements. The fuel clad or covering must withstand corrosion and thermal distortion throughout an acceptable operating 
range. There must also be sufficient heat transfer between the fuel elements and the coolant. All components must be able to withstand anticipated stresses without being appreciably distorted. If these materials will only withstand certain stresses, these will determine the operating range. In choosing a coolant, it is important that it have characteristics that will provide as wide an operating range as possible.

Some of the important physical characteristics of a coolant are the boiling point, the freezing or melting point, specific heat, thermal conductivity, and viscosity. The ideal coolant must have a high boiling point to function properly without undue interference caused by boiling.

The freezing or melting point must fall below the lowest operating ranges. A low freezing point prevents the possibility of solidification during reactor shutdowns.

The specific heat is the amount of heat required to raise a unit mass of a substance one degree on a temperature scale at either constant pressure or volume. It should be apparent that a material having a high specific heat will be able to remove more heat from a reactor than one with a low specific heat. If the coolant has a high specific heat, it requires more heat to raise the temperature one degree, and more heat can be transferred by a given mass of coolant.

The thermal conductivity plays an important part in heat removal. Whereas the specific heat is concerned with the ability of a material to absorb heat while resisting internal temperature changes, the thermal conductivity involves the ability of materials to conduct heat away from other substances. The ideal coolant readily conducts heat away from the fuel elements.

The viscosity of the coolant is of considerable significance in all forced-convection systems. The ideal coolant requires only a small force to be moved through the reactor and heat exchanger. Low viscosity in a coolant means that more coolant can be moved with smaller pumps at lower cost. When less pressure is required to circulate the coolant, proportionately less stress is placed on piping systems.

In addition to the se factors, the coolant must be relatively stable when subjected to high temperatures and to radiation fields. Both light and heavy water break down into their individual components when subjected to radiation fields. Since hydrogen and oxygen can form an explosive mixture, inert atmospheres must be maintained when substances composed of these elements are used for coolants. The inert atmosphere carries the potentially explosive mixture away from the reactor to some point at which recombination may occur. Particular attention must also be directed to the corrosive effect of the coolant upon various reactor components. 
As the coolant passes directly through the reactor core, it is imperative that the elements comprising the coolant have low cross sections for neutron absorption. An ideal coolant will have little or no effect upon neutron balance. Finally, the coolant should not be hazardous to operating personnel. If the coolant is of a corrosive, toxic, or radioactive nature, special measures must be used to protect the personnel. As has been stressed previously, it is difficult to find ideal materials for use in reactor construction; thus, there is no perfect coolant for removing the heat produced in a reactor.

\subsection{Reactor Coolants}

Reactor materials used for coolant purposes may be divided into four general groups, namely, gases, ordinary and heavy water, organic liquids (hydrocarbons), and liquid metals.

When the reactor field was in its infancy, cooling was achieved by forcing air through the reactor with fans and then venting the gas to the atmosphere. This procedure was simple, and a complete closed circuit was not required. In addition, air was readily accessible, easily handled, and nonhazardous. In today's high-power reactors, and in reactors with relatively high operating temperatures, air is unsatisfactory as a coolant because of its chemical activity and poor conductivity. Purely from the point of heat removal, hydrogen would be the ideal coolant. This element is highly unsatisfactory, however, as it forms an explosive mixture with air and, in addition, causes certain metals to become brittle after prolonged contact.

The next choice in the group of gaseous coolants is helium. Helium is an inert or nonreactive gas, with a very small cross section for neutron absorption, and indeed is sometimes used in small quantities in certain reactor systems. As a coolant it is not available in sufficient quantities and is too expensive for general use in a reactor.

Probably the most practical gases to consider as coolants are nitrogen and carbon dioxide. Both have been used as coolants. The primary disadvantage in using any gas for this purpose is cost. Unless a gas is under a fairly high pressure, the cost of pumping it through the system becomes considerable. This is an important aspect of reactor economy.

Both ordinary water $\left(\mathrm{H}_{2} \mathrm{O}\right)$ and heavy water $\left(\mathrm{D}_{2} \mathrm{O}\right)$ are good coolants insofar as their heat-removal properties are concerned. These compounds serve not only as coolants, but as moderators as well. Ordinary or light water is inexpensive, easily pumped, has a high specific heat, and has fairly good properties of thermal conductivity. In opposition to these advantageous properties are a relatively high neutron-absorption cross section, a low boiling point, and the fact that water is corrosive and tends to decompose at high temperatures. 
Heavy water exhibits many of the same properties of ordinary water but has one outstanding advantage, a very low cross section for neutron absorption. The cost of this compound is considerable, however, being approximately twelve dollars per pound. The relatively low boiling points of both ordinary and heavy water usually requires pressurization of the system in reactors where high temperatures are anticipated.

When temperature requirements make the use of water impractical for cooling purposes, liquid metals may be considered. The best of these is sodium, which possesses excellent heat-removal characteristics. Sodium does not require pressurization at higher temperatures, is quite stable, and attacks reactor metals to only a slight extent. A disadvantage of sodium is its high reactivity with the oxygen in air and water, and extreme caution must be exercised in its use. Further, sodium becomes highly radioactive when passed through a reactor. This requires special shielding materials for this type of system. In fast reactors, sodium is practically the only coolant that is at all satisfactory.

To compromise between water, which exhibits certain undesirable characteristics and pressure requirements, and sodium, which is so chemically active and hazardous, consideration is being given to certain of the organic compounds, such as the polyphenols. These organic structures do not require pressurization, are fairly stable under heat and radiation stresses, and are not corrosive. The heat-removal qualities of these compounds, however, are definitely inferior to those of water and liquid sodium. In choosing a coolant, emphasis again is placed upon the compromises necessary to achieve the best method of heat transfer for a particular reactor. Reactors currently operated by the Reactor Operations Division at Argonne National Laboratory use light and heavy water for the primary coolants.

\subsection{Varied Methods of Reactor Control}

The control mechanisms are important aspects of reactor construction. Controls are necessary (1) to raise the reactor to some operating power, (2) to maintain any predetermined power level, (3) provide rapid shutdown whenever necessary, and (4) to prevent the chain reaction from becoming violent. Understandably, if the multiplication factor were always equal to unity, the number of available neutrons causing fission could not increase from generation to generation, and the system would be very inflexible. A reactor must contain some material capable of changing reactivity in order that the multiplication factor ( $k$ ) may exceed unity and thereby allow an increase in power. This material must also function as a control capable of decreasing the value of $k$ to less than unity in order to provide for plant shutdowns. Further, controls must be capable of halting neutron growth and preventing too rapid a liberation of energy. 
Methods of reactor control involve the addition or subtraction of materials. The positioning of these materials serves to disrupt the neutron balance, which changes the value of $k$.

A reactor that is shut down has a multiplication factor less than unity. The value of the reactivity at shutdown is a function of the fuel load, neutron absorption and reflection, environmental conditions such as temperature and in-pile experiments, and the presence of fission product poisons and burnable poisons. To start up a reactor, this reactivity state must be changed. Generally speaking, some form of mechanical motion in or around the reactor core is required. Reactivity changes may be made by manipulation of fuel components, moderator, reflector, and neutronabsorbing types of control rods.

Increase or decrease of the amount of fuel present in a reactor changes reactivity. When additional fuel is added, more atoms are present to maintain successive generations. Conversely, fuel depletion causes a decrease in reactivity. During the lifetime of a fuel loading, the fissionable material is used up. This means that the number of fissions will decrease; consequently, $k$ will be reduced, and movable control rods must be moved further from the core to compensate for the reduction in $k$. The addition or removal of moderator and reflector materials also alters the value of $k$. The moderator functions to slow down fast neutrons to the thermal range, thereby enhancing the likelihood of fission. When moderator is removed from the system, less slowing down of neutrons occurs, and fewer slow neutrons are available to cause fission. Alteration of the structure of the reflector is another method of reactor control. When reflector materials are removed more leakage occurs, fewer neutrons are directed back into the core, and reactivity decreases. Reactors have been constructed with rotatable cylinders mounted at the corners of the core and in the reflector region. Parts of the cylinder circumferences are made of reflector materials, and the other parts of neutron-absorbing material. The reactivity of the core is changed as a function of the rotational position of the cylinders.

The importance of core geometry in maintaining a neutron balance should be recalled. By shifting the fuel to different geometric arrangements, more or less leakage will occur, and the neutron balance can be altered in the desired direction.

\subsection{Operation of the Control Rods}

It is important for an operator to understand fully how the control network functions and what its capabilities are. The operator is an integral and important part of any reactor, and certainly an extension of reactor control mechanisms. In constructing a reactor, however, it is not advisable to rely entirely upon human operation, as no individual is infallible. For 
this reason, all emergency safety actions are failsafe and fully automatic. In addition, complete manual control of a reactor is tedious and requires great alertness and training on the part of the operator. In changing the power level, skill and anticipation are required to prevent overshoot of the power level. Poisoning and temperature effects create a need for periodic manipulation of control rods. The automatic systems do an excellent job and also eliminate some of the work load of operators. This does not diminish in any way the importance of the operator. A mechanical or electronic component serves a definite purpose, but the operator must make most of the decisions.

At this point, the role of the control rod should be examined in some detail. Recall that the production of neutrons minus the leakage minus the absorption is equal to one for criticality. If we wish to alter the production of neutrons, we may alter some of the materials previously discussed, or change either the leakage or the absorption. To change the leakage, a window or hole might be cut in the reflector. A procedure of this type is rather time consuming and more or less permanent. The commonly accepted procedure is to change the multiplication by absorption. To accomplish this, control rods of various high cross sections for thermal-neutron absorption may be used to absorb neutrons from the reaction. It will be assumed here that the control rods are of the absorptive type only.

The rods may be moved in and out of the reactor individually or in banks. Certain groups of rods may be designated as safety rods and others as shim rods. Shim rods function to provide coarse changes in reactor power. Another rod, called the regulating rod, provides a fine control for reactor power.

It should be obvious that a multiplication factor $k>1$ is built into every reactor. Reactor control is accomplished by adjusting the movable absorbers in the core. A complete insertion yields a subcritical operation. To obtain criticality, the absorbers are withdrawn until $\mathrm{k}=1$. Further withdrawal from the critical position yields a supercritical condition.

Examine the method of changing power level in a simplified reactor by movement of control rods. Assume that the reactor is critical at a very low power level and that demands call for a power increase. If a control rod is extracted from the core by a small amount, the multiplication factor $k$ is changed from $l$ to a number slightly larger than one. The neutron level begins to rise. As the power rises to some previously determined level, it is obvious that the control rod must be inserted to the point where $\mathrm{k}=\mathrm{l}$. We have, then, after the power level becomes stable, a system where the power level is independent of the rod position. 
To change the power level the rod is moved in or out of the core area and then returned to a position at which $\mathrm{k}$ is again equal 1 . In this manner a reactor is controlled and a power level maintained.

\subsection{Control Rod Materials}

Ideally, the best absorber material for control or safety rod construction would be one with a high nuclear cross section for all energies of neutrons. Unfortunately, no such material exists. Following the theme of compromise in reactor structural components, materials having high absorption cross sections in the thermal range are used. Although the nuclear cross section is of primary importance in selection of a suitable material, physical considerations are also examined.

A control rod must have good mechanical properties so that it may be easily constructed and machined. The rods must also be able to withstand shock stresses. They must be resistant to corrosive effects, or at least capable of being alloyed with a material that is corrosion resistant. In addition, a safety-rod material must have good radiation-damage characteristics. This means that the rods must be resistant to swelling and distortion under neutron bombardment. A desirable poison material for control rods would be one which reacts with neutrons by means of the $(n, \gamma)$ reaction rather than the $(n, \alpha)$ reaction. In such reactions, a neutron is captured or absorbed and the compound nucleus, depending on its type, emits a gamma ray or alpha particle. The alpha particle being heavy and having a short range in materials produces brittleness, deformation, and swelling in certain reactor components. The damage caused from gamma radiation is not as great. Whenever possible, the material chosen for control rod use should have a low atomic number and a high density. This combination will yield a large number of absorbing atoms per volume.

Cadmium is used as a control and safety-rod material, but its use is generally restricted to research and low-temperature reactors. The element has a good cross section for neutron absorption, is inexpensive, but has a low boiling point (around $310^{\circ} \mathrm{C}$ ) and alloys poorly.

Boron-10 is another commonly used material. Although the isotope has a low atomic weight, its absorption cross section is excellent. The main disadvantage in the use of this material is the deleterious effect of the $\mathrm{B}^{10}(\mathrm{n}, \alpha) \mathrm{Li}^{7}$ reaction in producing radiation damage to components. Generally, only low concentrations of boron may be used in a control rod, although boron carbide and alloys of boron-steel have proved to be successful in this application.

Hafnium is another material that has been used in control rods. With this element absorption takes place by five successive ( $n, \gamma$ ) reactions. Hafnium has a good cross section for neutron absorption, fair 
resistance to radiation damage, and good resistance to corrosive effects. Some other materials that might be considered for control rod usage are gadolinium, nickel, iridium, and europium.

\subsection{Control Rod Nomenclature and Requirements}

The control rods may be classified in three groups according to function: safety rods, shim rods, and regulating rods. Each type of rod usually has a different speed of motion. The regulating rod maintains a constant desired power level by compensating for small reactivitychanges. This rod may also be used to assist in changing the power level from one state to another. The control aspects and speed of this rod are variable, but generally the worth of this rod does not exceed the delayed neutron fraction. Speed is regulated to provide swift movement both in and out of the core region. This movement provides a quick response for changes affecting the reactor.

As was previously mentioned, the shim rods are used as coarse controls for greater changes of reactivity than may be controlled by the regulating rod. The shim rods bring the reactor close to the desired power level. Shim rods are of particular importance in reactor startup, and their speed is generally quite slow. At the CP-5 reactor, the maximum speed of the shim rods is only one degree per six seconds. Further restrictions are placed on these rods in that only one may be moved at any given time. These rods must have a large reactivity worth.

Safety rods on the other hand are for "scramming" or rapid shutdown, and their motion must be very fast when being inserted. The worth of these rods must be appreciably greater than all of the excess reactivity within the reactor.

It should be apparent that different mechanical motions may exist for each of these three types of rods. In many reactors the functions are separate, and in others combined. At startup, the shim rod may be operated at a slow, normal speed. As soon as the rod is withdrawn from the core, its function may be changed to that of a safety rod. With the exception of certain types of regulating rods, all rods will have a provision for fast insertion into the core.

We have discussed briefly the "worth" of different types of control rods. Often the term "dollars worth of reactivity" is used. A group of control rods may be said to be worth X dollars. This term refers to the interval between delayed critical and prompt critical and is defined as

$$
\$=\Delta \mathrm{k} / \mathrm{v} \beta \quad \text {, }
$$

where $\Delta \mathrm{k}=\mathrm{k}-\mathrm{l}$ and $\beta$ is the delayed neutron fraction. The quantity $\mathrm{v}$ represents a number close to unity and is sometimes neglected for this 
calculation. Actually, $v$ represents the relative effectiveness of delayed neutrons in producing fission as compared with prompt neutrons. To illustrate this further, an example will be given. In a uranium-235-fueled reactor, for which $\beta=0.0068$ and $v=1.05,50$ cents of reactivity worth would represent the following effective multiplication factor:

$$
\$=\Delta \mathrm{k} / \mathrm{v} \beta \quad \text {. }
$$

Thus,

$$
\Delta \mathrm{k}=\beta \$ \mathrm{v}
$$

Therefore,

$$
\Delta \mathrm{k}=0.0068 \times 0.5 \times 1.05=0.00357
$$

In other words, an excess reactivity of 50 cents represents an effective multiplication factor of 1.00357 for this reactor.

\subsection{Poison Effects on Reactivity}

It has been indicated that control rods, fuel depletion, and temperature affect the multiplication factor of a reactor. Fission products, in-pile experiments, and burnable poisons also have an important effect on the neutron balance. As a reactor operates, fission products are created from the fuel. Many direct products exist, and in addition daughter nuclides are created by decay from these products. Many fission products, both direct and indirect, have high cross sections for neutron absorption and therefore may act as reactor poisons. Xenon-135 and samarium-149 are of particular importance because of their high absorption cross sections. As these products increase, control rods must be moved to compensate for their presence.

In-pile experiments also alter the multiplication factor. Experimental equipment often contains materials which act as reactor poisons. If care is taken when such experiments are inserted into the reactor, reactivity may be controlled so that no excessive changes occur.

Finally, poisons may be built into a reactor that, after constant bombardment, become supersaturated with neutrons. These materials are said to "burn up." As fuel decreases, the absorbing power of the poison decreases, and the two situations will counteract each other. In using methods of this nature, it may be possible to operate the reactor for longer periods of time without fuel change, thereby increasing reactor efficiency. 
CHAPTER III

\section{AUXILIARY SYSTEMS}

\subsection{The Cleanup System}

In the discussion concerning moderators and coolants, it was noted that certain impurities in both ordinary and heavy water caused a detrimental effect upon reactivity. It is possible to minimize this effect by maintaining a high level of purity throughout the reactor water systems.

Impure water contains many different minerals, oxides, and ionic materials, some of which affect core reactivity in much the same manner as in poisoning by fission products. When subjected to a neutron field, many of these materials become intensely radioactive. As the particulate and dissolved materials travel throughout the reactor system, they must be kept at a minimum, or additional shielding will be required.

Water decomposes to a certain extent under radiation. As the purity of the water increases, the rate of decomposition decreases. By keeping the rate of hydrogen formation at a minimum, a potentially dangerous explosion hazard is eliminated.

The purity of the water also determines the rate of corrosion. If a high degree of purity is not maintained, the rate of corrosion increases.

Normally, reactor water undergoes an extensive purification process prior to any nuclear application. The operating problem is to maintain this initial purity. Some degree of contamination occurs any time the reactor system is open, regardless how short this time may be. When water comes in contact with air, contamination occurs. Further, the introduction of new reactor components also causes contamination. Items such as new pumps, piping, valves, and even fuel elements introduce impurities upon insertion. These components are always thoroughly cleaned, but it is imposible to maintain complete cleanliness.

Obviously, the purity of reactor water may be partially controlled by insuring that this purity is of an acceptable value prior to being put in the reactor. To maintain the initial quality, a bypass cleanup system is used in conjunction with a closed primary system. A percentage of the primary system flow is diverted into this separate system. Eventually, as time passes, all of the primary water will flow through the cleanup system, and thus undergo additional purification. The cleanup system contains filters to remove particulate matter from the water system, and ionexchange columns which remove dissolved impurities that are present.

The general cleanup system consists of a filter, or series of filters, followed by an ion-exchange column and additional filters. The filters used 
are designed to remove particulate matter whose dimensions are of the order of $30 \mu(0.003 \mathrm{~cm})$. The use of these prefilters removes most of the loose particulate matter present in the reactor water. An ion-exchange column is also placed in the system following the filters. The column serves a twofold purpose. Primarily, the column functions to remove dissolved impurities, but the unit also removes some of the smaller solid matter not previously trapped in the filters.

To understand how the ion exchange works, let us consider certain chemical facts. Some atoms may gain or lose electrons. In doing this, they acquire an electrical charge. For example, a sodium atom may give up the single electron in its outer shell. When sodium has done so, it is left with one more proton than it has electrons and hence has a positive charge of one:

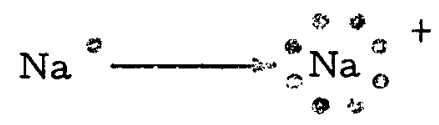

+1 electron

atom

We call such a charged atom an ion, in this case a sodium ion. A chlorine atom, which has seven electrons in its outer shell, may gain an electron. It becomes a chloride ion with a negative charge:

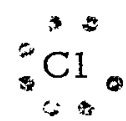

atom

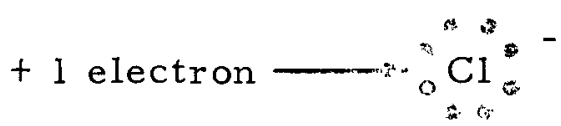

ion

A positively charged ion is called a cation and a negatively charged ion an anion. Pure water is a very poor conductor of electricity, but certain impurities in water make it a good conductor. These substances are called electrolytes. It will suffice to say that many impurities in water are ionic and that the movement of these particles results in a transfer of electrical charge, hence the name cation and anion. Positively charged cations such as sodium are attracted by the cathode, and the negatively charged anions to the anode. These facts may be related to the ionexchange column.

It is known that the dissolved impurities in the reactor water system are ions, i.e., particles charged either positively or negatively. The properties of these ions enable their removal from the reactor water system by passing the reactor water through a system which will remove impurities. Many high polymers, both natural and synthetic, will absorb either cations or anions. For example, cellulose in the form of cotton will remove ferric ions from solution. For reactor use, a judicious selection of these polymers is made, and formed into what is called a "mixed bed" resin. One type of resin removes cations, and another anions. 
The ion-exchange column is essentially a cylindrical tank filled with the mixed-bed resins. As the reactor water passes through the column, the ions are in intimate contact with the resins and are absorbed by them. By use of the proper resins it becomes possible to maintain water purity almost at the level of distilled water. The resins are restricted to a limited lifetime and must be replaced as they become spent. The ions build up to such an extent that the resins become supersaturated and no longer function properly. After the re-purified water leaves the ion exchanger, it passes through a second filter, prior to re-entry into the reactor. The second filtering system removes any grains of the resin that have been washed out of the column and prevents their entry into the reactor tank.

\subsection{Measurement of Water Purity}

Once the cleanup system has been installed and is functioning, some method of monitoring its effectiveness must be devised. In placing the measuring devices, one will normally measure the water purity as the liquid enters the cleanup system. This measurement occurs prior to any purification and essentially denotes the condition of all water in the reactor. To determine the effectiveness of the cleanup system, the purity is also measured as the water leaves the cleanup system.

One method of determining the purity of a water sample is to measure the acidity or alkalinity of the water. This alkalinity or acidity is dependent upon the concentration of the hydrogen ion. This value is designated by the symbol $\mathrm{pH}$ and is the value of the exponent of the molar concentration (omitting the minus sign). The $\mathrm{pH}$ of a neutral water solution is seven, meaning that in one liter of water the concentration of the hydrogen ion is $1 \times 10^{-7}$ molar. In using this method of representation, we may construct a table such as Table 3.0 .

Table 3.0

RELATION OF pH VALUE TO H ${ }^{+}$AND OH ${ }^{-}$CONCENTRATIONS

\begin{tabular}{llll}
\hline Molar Concentration of $\mathrm{H}^{+}$ & $\mathrm{pH}$ & $\begin{array}{c}\text { Molar Concentrations } \\
\text { of } \mathrm{OH}^{-}\end{array}$ \\
\hline 1 & $=10^{0}$ & 0 & $10^{-14}$ \\
0.1 & $=10^{-1}$ & 1 & $10^{-13}$ \\
0.01 & $=10^{-2}$ & 2 & $10^{-12}$ \\
0.0001 & $=10^{-4}$ & 4 & $10^{-10}$ \\
0.0000001 & $=10^{-7}$ & 7 & $10^{-7}$ (neutral water) \\
0.0000000001 & $=10^{-10}$ & 10 & $10^{-4}$ \\
\hline
\end{tabular}


It should be apparent that as the acidity of the water decreases, the $\mathrm{pH}$ value (by definition) increases. Any solution with a $\mathrm{pH}$ value greater than seven, is basic or alkaline.

If the concentration of hydrogen ion, denoted as $\left(\mathrm{H}^{+}\right)$, is not an integral power of ten, the following definition of $\mathrm{pH}$ may be used. $\mathrm{pH}$ is the negative logarithm of the hydrogen ion concentration. The $\mathrm{pH}$ meter is calibrated in positive numbers and fractions with seven as the neutral point. In using a table such as Table 3.0, it is possible to calculate the hydrogen ion concentration at any given time.

The measuring device itself consists of two units, an electrode as sembly and an external voltage-measurement component. The electrode assembly is immersed in the solution to be measured and a voltage is produced between the faces of the two electrodes. This voltage signal is sent to a meter and converted to a representative pH reading. Generally, a third probe is also inserted into the solution to compensate for temperature changes and give a more accurate reading.

\subsection{Conductivity Measurement}

In discussing the ion-exchange column, it was observed that many of the impurities found in the reactor water system are ionic. If two oppositely charged electrodes are placed in a solution containing ions, the positively charged ions will be attracted to the negative electrode, and the negatively charged ions will be drawn to the positive electrode. This movement of charged particles through the solution results in a transfer of electrical charge, and the solution is said to be conducting an electrical current. This type of conduction, which results from a movement of ions through a solution, is called electrolytic or ionic conduction to distinguish it from metallic conduction, which occurs as electrons flow through wire. The amount of current transferred is dependent upon the number of ions present in the solution.

Water varies in its ability to conduct a current. This variance is dependent upon the number of ions present in the solution. Absolutely pure water offers a resistance of approximately twenty megohm centimeters. This value is the resistance measured between opposite faces of a one-centimeter cube of water. As purity of the water decreases, the resistance also decreases.

The conductivity meter measures the resistance of the water, and this resistance is then related to the purity. The instrument consists of a probe with two platinum electrodes. The probe is immersed in the solution, and the resistance is measured between the two electrodes. The conductivity meter itself is read either in units of resistance (megohm centimeters), or in units of conductance (micromhos per centimeter). 


\subsection{Helium System}

Voids exist above the surface of the water within the reactor vessel. Air cannot be allowed to fill these open spaces for several reasons. Air is a mixture of gases, some of which become radioactive. If air is used for a reactor atmosphere, the shielding and problem of personnel protection become considerable. The presence of air over the reactor also increases the rate of corrosion. Nitric acid is formed from the nitrogen in the air and the decomposed water. Hydrogen in air also increases the corrosion rate. Finally, it is desirable to sweep away the decomposed water either to a recombination system or to a disposal facility. For these reasons, a helium atmosphere is generally provided to cover the reactor system. This atmosphere is not limited just to the reactor vessel, but occupies any space above the water-storage tanks and all voids in various piping components.

Helium is used for this purpose because it exhibits many desirable properties. The element is one of the inert gases, which means that helium will not undergo chemical reactions. The element does not absorb neutrons and has no effect upon reactivity. Helium is twice as heavy as hydrogen but has excellent lifting power. This property allows the gas to pick up the dissociated materials, and, as helium is easily pumped, to carry these materials away. The gas itself does not become radioactive and is nonhazardous to operating personnel. Helium used in a reactor system must be of a very high purity, and the cost of the gas is considerable. The definite advantages, however, more than outweigh the relatively high cost.

Another purpose of providing a helium atmosphere is to prevent an undesirable entry of air into the reactor system. To accomplish this, the helium system is pressurized to a value slightly greater than atmospheric pressure. This differential pressure is approximately equal to the force exerted by 2 in. of water and is maintained by a suitable gasholder. This positive pressure with respect to atmosphere prevents air from entering the system in the event of a leak.

The gasholder itself is simply a device capable of varying its internal volume in such a manner as to maintain a constant positive pressure throughout the system. Two different types of gasholders will be considered, both of which have been used in Argonne reactors.

At the CP-5 reactor, the gasholder is a cylindrical tank in which a diaphragm or disc moves within the tank in a vertical plane (see Figure 3.1). The disc is usually quite heavy and counterbalances are adjusted to support this weight. The disc moves automatically to maintain a constant pressure. 
The gasholders used at the Janus and Juggernaut reactors are of a different nature and consist of two drums, one inverted inside the second (see Figure 3.2). An oil seal is provided between the two drums, and the movable section adjusts itself within the confines of this seal to maintain the desired pressures.

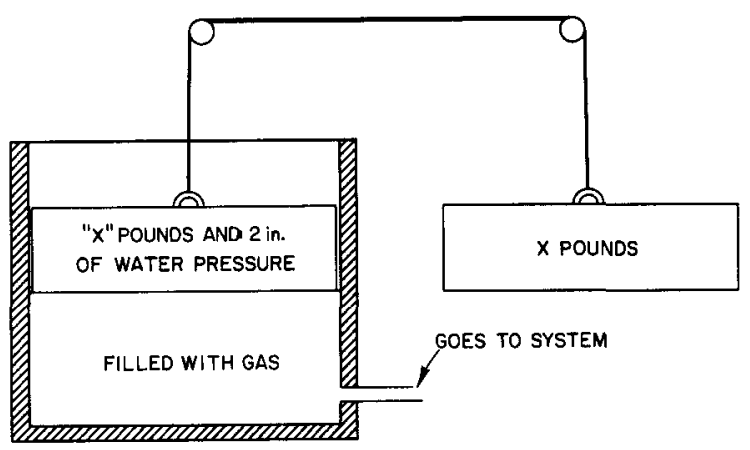

Fig. 3.1. Disc-type Gasometer

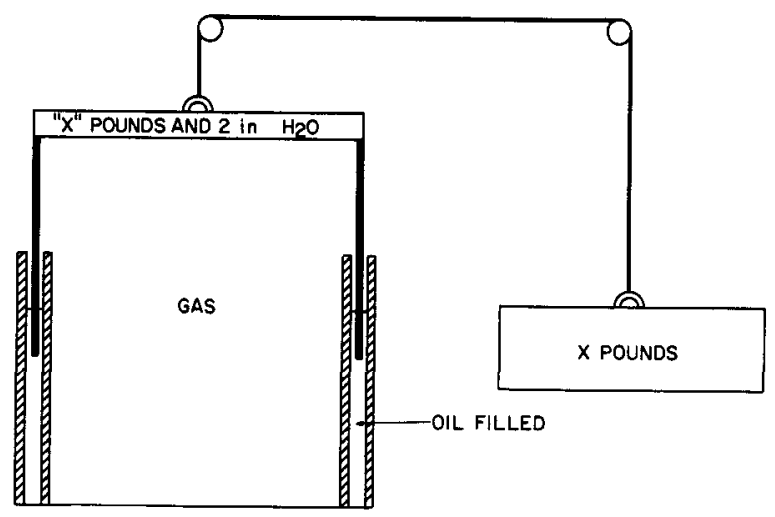

Fig. 3.2. Oil-seal Gasometer

In both types of gasholders, the movable components maintain a constant pressure upon the helium system. The helium system normally contains a safety blow-off valve to prevent overpressurization. The inlet to the helium system is placed at some appropriate position in the gasholder and flow originates from the gasholder.

\subsection{Catalyst Chambers}

In water-moderated-and-cooled reactors a special problem exists with the helium system. Water dissociates to some extent under radiation. In reactors in which ordinary water is used, the problem is in removal of the hazardous products. These may either be recombined or disposed.

Heavy water, however, is much too expensive to throw away and must be recombined. To accomplish this, a catalyst chamber is installed at some point in the helium system. The chamber is filled with palladiumcoated aluminum oxide pellets. The palladium-coated pellets are the actual catalytic agent. (A catalyst is a substance which alters the rate of a chemical reaction without becoming permanently changed itself.) In this application, the pellets speed up or enhance the recombination of either ordinary or heavy water. The helium gas is passed through the chamber and any dissociation products are recombined, recovered, and then returned to the main water system.

Occasionally, the helium system becomes contaminated by various gases, including air which has managed to enter the system. Frequent ana1ysis of helium gas samples is made and when conditions warrant, the entire system is purged. The atmosphere is completely replaced by pure helium and the contaminated gas discharged to a ventilation system. To recover any heavy water vapor, cold traps are usually placed in the purging lines. 
CHAPTER IV

REACTOR SHIELDING

\subsection{Biological Shielding}

In a general reactor scheme, a biological shield surrounds the reflector zone. This shield serves the purpose of attenuating the radiations emanating from the core. Normally, a shield is a combination of materials which attenuates both neutrons and gamma rays. The reactor shield is necessary to reduce exposure of personnel. The problems of radiation protection are of considerable importance because of the intensity of the radiations found around reactor sites. The alpha, beta, gamma, and X rays emitted by various nuclides within a reactor, and the neutrons that escape from the core are all known to have detrimental biological effects upon the human system. The harmful consequences of these radiations are in proportion to their relative ionizing power. This ionization causes orbital electrons to be ejected from atoms comprising various compounds in the body. It has been determined by biologists that the radiations affect individual cells, possibly by destroying enzymes which determine the particular function of a given cell. High dose rates of radiation will produce severe surface burns in addition to the cell damage which occurs within the body. If accidentally inhaled, certain particle emitters tend to accumulate in certain sections of the body, and their harmful actions continue even though the individual may no longer be near a radiation source. A sufficiently high amount of radiation has a lethal effect upon the biological system. All of these types of radiations exist at unprotected irradiation facilities. The rapid pace at which atomic energy projects are developing makes it necessary for a large number of personnel to study and work with components involving radiological hazards. The reactor shield protects these people.

\subsection{Experimental Requirements}

In a research reactor, experimental facilities are established to obtain needed data. Much of the experimental equipment depends upon the intensity of the radiations measured. This intensity is proportional to the square of the distance from the source. To obtain required intensities, experiments quite often must be conducted in close proximity to the reactor. This indirectly imposes an additional shielding requirement. Primary counting instruments normally count very low levels of radiation, even though the actual intensity may be quite high. In designing a shield, materials are chosen to provide maximum protection to personnel with a minimum thickness. The shield must prevent biological damage and still be flexible enough to satisfy experimental requirements, both for intensity of the source and radiation background limits. 


\subsection{Radiations Produced in Fission}

A mass of uranium-235 undergoing fission yields many different types of particles, both charged and uncharged. This discussion will be limited to only the main particles, with the understanding that many other particles do exist. In the group of charged particles found within a reactor are the fission products, alpha particles, beta particles, protons, positrons, and deuterons. The uncharged radiations of concern are the neutron and the gamma ray.

To examine certain properties of some of these particles, we place a small quantity of a material containing radium at the bottom of a hole bored into a piece of lead (see Figure 4.0). All radiations will be stopped

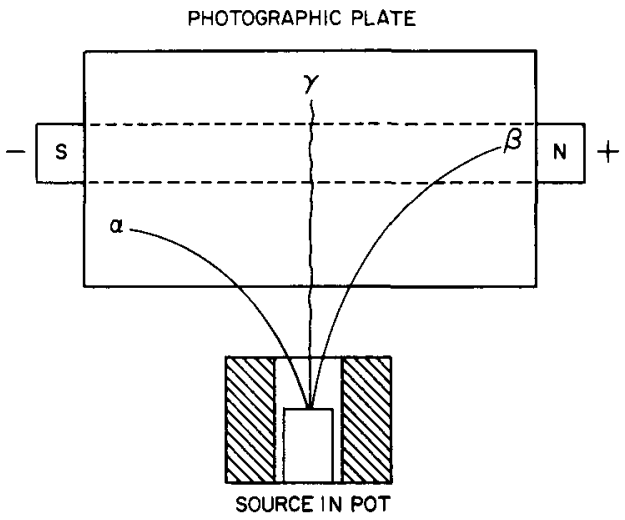

Fig. 4.0. Deflection of Radiation in Magnetic Field by the lead except those headed directly upward. The sample, enclosed in its lead shield, is placed between the poles of a magnet (see Figure 4.0) with a photographic plate positioned above it to receive and detect any rays that are emitted. In. observing the direction of the emissions, it will be found that one set corresponds to what one would expect for relatively heavy, positively charged particles passing through a magnetic field. These are alpha particles, and further study proves them to be helium nuclei. Other particles are deflected in the opposite direction and to a much greater extent. These are the beta rays. They behave like negatively charged particles of very small mass. These particles are electrons. The third ray, which strikes the center of the photographic plate, is not deflected at all. This is the gamma ray, which is an electromagnetic wave similar to light but of a shorter wave length.

The speed of the gamma rays is the same as that of light or $3 \times 10^{10} \mathrm{~cm} / \mathrm{sec}$ and equal to $186,000 \mathrm{miles} / \mathrm{sec}$. The speeds of the alpha particles depend on the kind of nucleus from which they are emitted, but ranges are found from approximately 9,000 to $14,000 \mathrm{miles} / \mathrm{sec}$. Beta rays often travel as fast as $100,000 \mathrm{miles} / \mathrm{sec}$.

Alpha particles can penetrate only the thinnest sheets of metal and are even stopped by an ordinary piece of paper. The beta particles have greater penetrating power than the alpha particles, but are stopped by approximately $0.25 \mathrm{in}$. of aluminum. Gamma rays can penetrate thick layers of metal and can be detected even after traveling through 8 or 10 in. of lead. The relative penetrating powers of these three types of radiation tested with aluminum are approximately as follows: alpha particles l; 
beta particles 100; gamma rays 10,000. An experiment of this type points out that the properties of various particles dealt with in the nuclear field are quite different. Because of this fact, a shield capable of stopping one type of radiation may be entirely unsatisfactory for any one of the other particles.

The fission products, alpha particles, deuterons, and protons are positively charged. These particles have a large mass and move with relatively low speeds. When particles of this type enter a shielding material, they disturb orbital electrons in the atoms in the material and in doing so lose some of their energy. The greater part of the energy of a particle is found in the form of kinetic energy or the energy of motion. A rough comparison of the positive charged particles versus other types of particles may be made. The shield may be compared to a netlike structure having a small mesh. The size of an alpha particle, relatively speaking, would be represented by a basketball when compared with a neutron or gamma ray. From this view, the neutron or gamma ray could be compared with a bullet, as these particles are very small and move at much higher speeds. The speed of the particle is of great importance when shielding is considered. When the basketball is thrown at the net, it will either bounce off or be stopped. A bullet, on the other hand, would pass right through the net, meeting little or no resistance. By analogy, the same type of events occurs when particles strike shielding matter. The heavy, slowly moving particles are readily stopped with a minimum of material, whereas other particles require additional shielding. Approximately 0.0005 in. of aluminum will stop any of the fission products, and $0.002 \mathrm{in}$. will stop alpha particles with an energy of $7 \mathrm{Mev}$. This means that the fission products, alpha particles, and certain of the other positively charged particles will be contained within the fuel cladding. The beta particle is much lighter in weight and moves with a higher speed. Beta radiation consists of free electrons. These electrons are easily captured and present no particular shielding problem. When dealing with any charged particle, we may assume that the normal thickness of any combination of fuel cladding, moderator, and reflector will be sufficient to provide adequate shielding for all radiations due to charged particles.

\subsection{Uncharged Particles}

The massive shields around a reactor are required to stop gamma rays and neutrons. A nuclear reactor is a profuse source of gamma rays. These rays are produced from several different sources. When a uranium235 nucleus undergoes fission, the fission is accompanied by gamma-ray emission, either as prompt gamma rays or as fission product gamma rays. An additional source of gamma radiation is the many radioactive neutroncapture processes which occur in the fuel, moderator, coolant, and structural materials of a reactor. In addition, the absorption of beta particles may also give rise to $X$ radiation. These emissions are ionizing radiations emanating from the reactor at tremendous speeds. The basic engineering problem is to construct a shield satisfying all requirements but still leaving the reactor as a flexible research tool. 
The absorption of gamma radiation can be attributed to three separate physical processes of interaction with matter. Each of the types contribute to the total absorption. The role each process has in absorption varies both with the energy of the radiations being absorbed and with the properties of the absorbing material. These three processes are known as (1) the Compton effect, (2) the photoelectric effect, and (3) pair production. Each of the three processes will be examined briefly.

The first of the three effects that account for attenuation of gamma radiation in matter is the Compton effect. This can be understood in terms of a collision of a gamma ray with a free electron. When speaking of free electrons, the term is used in a relative sense, as it deals entirely with atomic electrons. These electrons are bound to the atoms in the absorbing material. If the gamma-ray energy exceeds the atomic binding energy of the struck electron, the latter may be considered as a "free" electron. For the interaction of gamma radiation with energy of about 0.l Mev or higher, with electrons, the electrons may be considered to be free. This distinction becomes very important in computing the role of Compton scattering as an effective method of gamma-ray attenuation. (For gamma radiation of lower

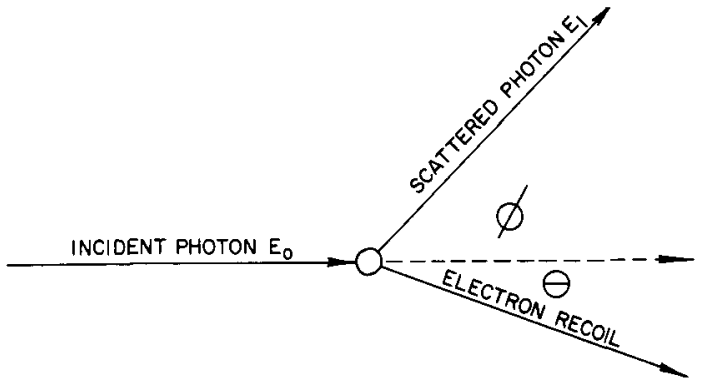

Fig. 4.1. The Compton Effect energy, the photelectric effect becomes much more important.) In Compton scattering, a gamma ray or photon enters the shielding material, striking an electron a glancing blow, the reby losing some energy (see Figure 4.1). This can be described as a collision between a gamma ray with an electron initially at rest. It should be noted that the gamma ray emerges with diminished energy $E_{1}$ scattered at an angle $\phi$. The electron moves away from the point of collision at an angle $\theta$ and carries away the energy lost by the gamma ray in the form of kinetic energy.

The second of the physical processes that lead to the attenuation of gamma radiation passing through matter is the photoelectric effect. This process results in the complete absorption of a gamma ray by an atom of the absorbing material, thereby causing an electron to be ejected with a reasonably high kinetic energy (see Figure 4.2). The photoelectric effect is the predominant type of interaction with matter of gamma rays having energies below $0.1 \mathrm{Mev}$. It is important to remember that in this case the gamma ray is

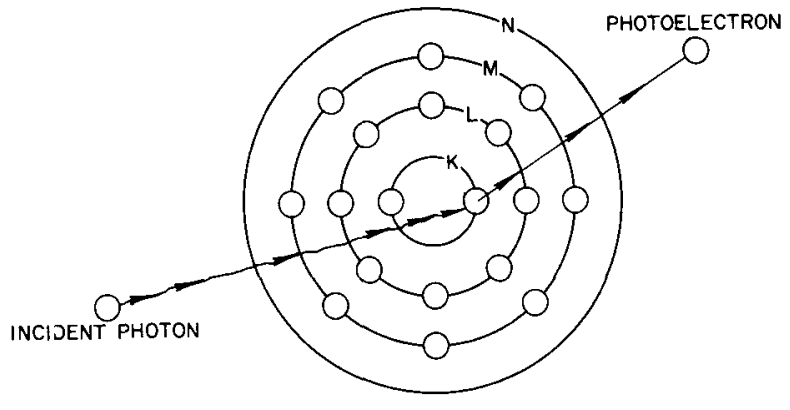

Fig. 4.2. Photoelectric Emission of $\mathrm{K}$ Electron 
completely absorbed. This happens as the gamma ray interacts with the atom as a whole, which carries away momentum as it recoils after the collision. The most probable reaction is one between the gamma ray and the most tightly bound electrons, i.e., the two $\mathrm{K}$-shell electrons. This shell is involved in about eighty per cent of the photoelectric absorptions if the incident gamma-ray energy is great enough to overcome the binding energy of the $\mathrm{K}$ electrons. Even though the gamma ray is completely destroyed in a photoelectric absorption, it should be understood that the removal of a $K$ electron will cause the emission of $X$ rays as the outer electrons fill the gap left in lower energy levels of the atom. As the gamma-ray energy decreases below the binding energy of the $\mathrm{K}$ shell, a sudden drop occurs in the interaction probability curve, and similar drops will appear as the energy falls below the binding energy of the L, M, etc., shells. As the energy of the gamma ray increases beyond the binding energy range of the $\mathrm{K}$ shell, the interaction probability also decreases.

The final mechanism that will be considered is pair production. In effect, this is the conversion of radiation energy into mass. This effect, which increases with increasing gamma energies, results in an incident gamma ray changing into two particles. A gamma ray is destroyed and an electron pair (an electron and a positron) are created (see Figure 4.3).

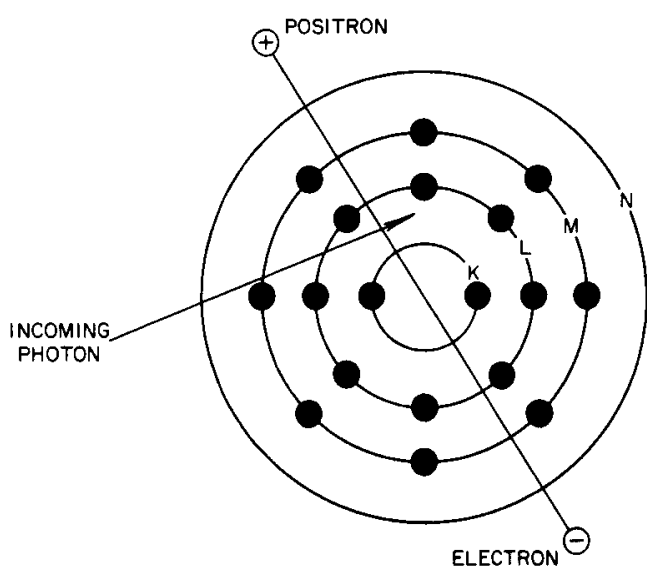

Fig. 4.3. Pair Production These particles may be called positive and negative electrons. This effect takes place primarily in the vicinity of a nucleus, and the created particles must have equal and opposite charges according to the principle of conservation of an electric charge. A gamma ray, with an energy equal to or more than $1.022 \mathrm{Mev}$, is converted to an electron carrying a positive charge and one with a negative charge. The figure $1.022 \mathrm{Mev}$ is twice the restmass energy of an electron converted to Mev.

Examine what happens to the newly created particles. Electrons have a negative charge, and present no particular shielding problem. The positive electron, or positron, is an example of what is called anti-matter (see Figure 4.4). A free electron is readily captured by ions as previously discussed. A secondary effect occurs when a positron collides with an electron. These two particles are attracted together, forming secondary gamma rays with an energy of $0.51 \mathrm{Mev}$, and the electron and positron disappear. Any absorbing material used for shielding must be capable of stopping both primary and secondary gamma rays. 
The materials used for a

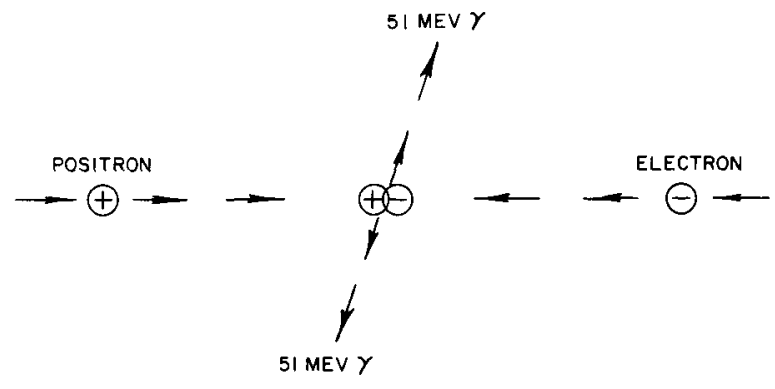

Fig. 4.4. Positron and Electron Interaction reactor shield employ all three of these processes for gamma-ray attenuation. The relative effectiveness of the three mechanisms is shown in Figure 4.5. The increase in the cross section for pair production with increase of gammaray energy is in direct contrast with the behaviour of the cross sections for the Compton and photoelectric effects, both of which fall off with increasing photon energies.

It is not implied that in the Compton scattering and photoelectric effects all attenuation ceases as gamma rays of higher energy are produced. Some attenuation occurs at all energies. The net effect, however, is diminished as the energy of the incident gamma is increased. The existence of these three processes is one of the phenomena which makes nuclear science so interesting. The problem becomes how to shield against gamma rays. Pair production absorbs gamma radiation of higher energy, and Compton scattering and the photoelectric effect eliminate the problem of absorbing radiations of lower energy.

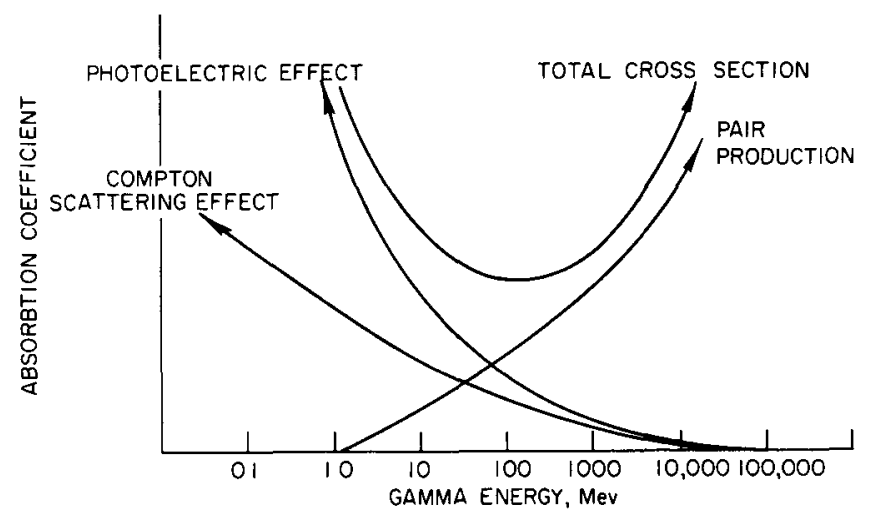

Fig. 4.5. Gamma-ray Attenuation

The other uncharged particle to be considered as a cause of difficult shielding problems is the neutron, which, as the name implies, is electrically neutral. Because of this property, neutrons have a very high penetrating power. When the neutron passes through various materials it is not scattered by electrons or nuclear electrical fields. It can only be removed by more or less direct collision with atomic nuclei. The cross section of any given material for neutron absorption is a measurement of the probability that an absorption will occur.

Before a neutron can be stopped, it must be slowed down. Moderators such as heavy water, carbon, or beryllium will effectively accomplish this. It is only after the neutron has been slowed down that it may be captured or removed. In addition to the moderator as a means of effectively slowing neutrons, mixtures of certain heavy elements and compounds containing hydrogen are often used. Heavy materials such as iron, lead, and bismuth do not necessarily capture neutrons, but will help in slowing them 
down to about $0.5 \mathrm{Mev}$. High-density concrete and other materials are used to absorb the slowed particles. The entire process then is two-fold: (1) neutrons are slowed down, and (2) slow neutrons are captured or absorbed.

\subsection{Shield Components}

Many different types of elements can go into the makeup of the completed reactor shield. Inasmuch as charged particles are easily captured or absorbed, essentially a shield is required to stop gamma and neutron radiations emanating from the reactor. The shield must perform three individual functions. It must slow down neutrons, capture the slow neutrons, and absorb gamma radiations. The discussion of moderators has shown that they effectively slow down neutrons. A part of the neutron shield, in the form of moderators, is the reactor itself. Such materials as iron, copper, cadmium, boron, and lithium have good cross sections for neutron capture, and combinations of these materials may be used in shield construction. The absorption of gamma rays is accomplished by using elements such as lead, iron, and even depleted uranium. Heavy and light water are good gamma-shielding mediums, and most heavy elements will accomplish gamma attenuation.

\subsection{Shielding Arrangement}

The actual shield usually consists of about 6 to $8 \mathrm{ft}$ of high-density concrete and required thicknesses of other suitable materials. Because of the shield's primary function for health protection, it is generally known as a BIOLOGICAL SHIELD. Through the absorption of neutrons and gamma radiation, that part of the shield which is in immediate contact with the core may become thermally hot. For this reason, special cooling facilities are normally provided to prevent cracking or other heat damage. For this reason, the inner portion of the shield, generally known as the THERMAL SHIELD, is usually constructed of steel plates. This THERMAL SHIELD

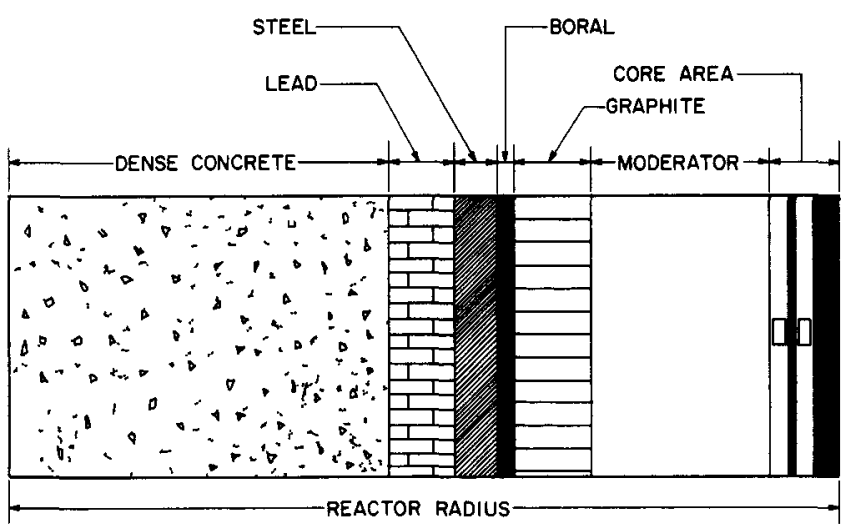

Fig. 4.6. Typical Shielding Arrangement may also be considered as two separate parts: the gamma shield and the neutron shield. Figure 4.6 shows a typical shielding arrangement, including the reactor vessel. The moderator is shown as part of the shield, as it was chosen to control neutron economy by slowing the fast neutrons. The graphite zone directly outside the vessel serves as a moderator-reflector.

It has been mentioned that such materials as iron, copper, 
cadmium, and boron have fairly high cross sections for neutron absorption. When thermal neutrons are absorbed, the atom involved liberates energy of about $6 \mathrm{Mev}$. This is a large amount of energy, and, as a great number of this type of absorptions occurs, it is necessary to pay particular attention to the manner and form of radiation removing this energy. The element iron has a high capture cross section for thermal neutrons, but almost all of the resulting energy is released by the emission of one highenergy gamma ray. If iron is to be used as a neutron shield, further shielding will be necessary to absorb this secondary gamma ray. Elements which will interact with neutron-gamma reactions are not as advantageous as those which undergo neutron-alpha-type reactions. By this it is meant that, as the neutron is absorbed by the shielding material, the resulting energy is given off in the form of an emitted alpha particle. Recall that alpha particles carry a positive charge and are relatively easy to shield. Boral, an aluminum-boron complex clad with a material suitable for stopping alpha particles, is such a compound. The neutrons are captured, energy is given off in the form of alpha particles, and these in turn are absorbed by the clad. One-half inch of boral shielding will comprise an excellent thermal neutron shield. It has already been mentioned that steel plates usually surround this portion of the thermal shield to prevent damage caused by heating.

To accomplish absorption of gamma radiations, a layer of lead is constructed adjacent to the neutron shield. Lead has a fairly good cross section for gamma absorption and, since it is the cheapest of the good gamma absorbers, has proven successful in this application. The final step is to encase the reactor shield in a layer of high-density concrete. Concrete of this type is usually filled with lead shot and/or iron punchings. The primary consideration after shielding requirements have been satisfied is to make the entire shield as thin as possible in order that experimental equipment may be placed close to the reactor.

To review, consider the steps necessary to complete the shielding arrangement:

1. Selection of moderator and reflector, both to conserve and slow down neutrons.

2. Selection of shielding materials for neutron absorption by means of neutron-alpha or neutron-gamma types of reaction.

3. Provision of extra structural support and cooling to prevent radiation and heat damage to the shield.

4. Selection of gamma-absorbing materials to stop gamma radiation coming from the reactor.

5. Provision of a shield casing of materials such as concrete to absorb any radiations that may possibly have escaped from the other shielding components. 


\subsection{Ir radiation Facility Shielding}

The research reactor is used for experimental purposes. This means that provisions must be made for facilities which will permit a release of radiation in predetermined amounts. Obviously, the only way to

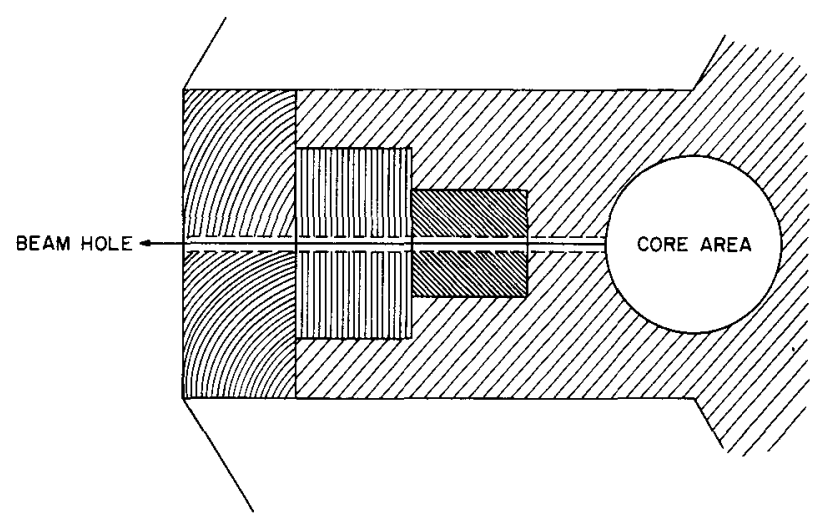

Fig. 4.7. Line-of-sight Shielding accomplish this is to disrupt the shield. When this is done, shield integrity is lost and leakage occurs. To seal up the experimental beam holes when they are not in use, "plugs" are used. The lineof-sight method (see Figure 4.7) is used to seal the beam holes.

Observe that the plugs and hole liners are stepped. Any escaping radiation would have to make at least two right-angled bends to escape from this type of shielding arrangement. Generally, if the source cannot be observed through annulus, radiations will be contained. The only restriction on this stepped arrangement is to insure that sufficient shielding remains to stop the radiations.

The operator will often be called upon to handle and remove samples from these reactor access ports. Operator assistance may also be required in the placement and removal of experimental equipment. Reactor personnel are working with radiation daily. THEY MUST NOT BECOME COMPLACENT! A shield is provided around the reactor for your protection. When it is necessary to work near or around radiation beams, the fundamentals of shielding should be recalled. Only a combination of shielding will stop all types of radiation. A lead brick will not provide a good shield against a neutron beam. Materials such as heavy concrete or mixtures of iron and paraffin are much better in this application. Absorbers such as boral, cadmium, and other s would be excellent, but it must be remembered that these materials suffer neutron-alpha reactions, neutrongamma reactions, etc., and other shielding may be required to stop the gammas. 


\section{CHAPTER V}

\section{REACTOR TEMPERATURE AND PRESSURE MEASUREMENT}

\subsection{Necessity of Temperature Measurement}

A nuclear reactor is an energy-producing scientific tool. It is important to realize that reactors do not in themselves produce mechanical or electrical energy; they produce heat. The effect of heat energy upon reactor characteristics has been discussed previously (see Sections 1.12 and 2.4). Heat is produced in the core, and it is necessary in reactor operation to measure the amount of power generated in the core region. A convenient method of determining reactor power level involves a temperature measurement. Thus, temperature-measuring devices are necessary in at least two locations in the primary cooling system. If a reactor contains supporting secondary systems, additional measurements become necessary. In short, temperatures tell reactor personnel much about the actual behaviour of the reactor. By using appropriate devices it is possible to obtain direct information regarding internal temperatures, fuel-element temperatures, shield cooling, primary and secondary system performance, ion-exchanger status, and many others.

\subsection{Temperature Scales and Units}

A change in the temperature of a substance will occur when energy is lost or gained by the substance. This change of temperature can be detected by an instrument such as the familiar thermometer, which is filled with a liquid whose density changes with temperature, or by a thermocouple whose electrical properties change with temperature.

For most common uses the familiar mercury thermometer is used. It has a bulb, filled with mercury, connected to a fine tube into which the mercury can flow as it expands when the temperature rises. The scale of a thermometer may be one of several types. In this country, the Fahrenheit scale is generally used for ordinary purposes, and the most common scale in scientific work is the Centigrade. On the Centigrade scale, the $0^{\circ}$ mark is the point where the column of mercury stands at the freezing point of water, and the $100^{\circ}$ mark is where it stands at the boiling point of water under one atmosphere of pressure. On the Fahrenheit scale, the freezing point of water is $32^{\circ}$, and the boiling point of water under one atmosphere of pressure is $212^{\circ}$. The relationship of the Fahrenheit scale to the Centigrade is shown in Figure 5.0 .

Sometimes temperature measurements are made in both systems of units, so it is necessary to easily convert from one unit of measurement to another. Consider Figure 5.0. A difference is noted in the number of units between the freezing and boiling points of water for each scale. In the Fahrenheit scale, there is a difference of 180 degress $\left(212^{\circ}-32^{\circ}\right)$, and in the 


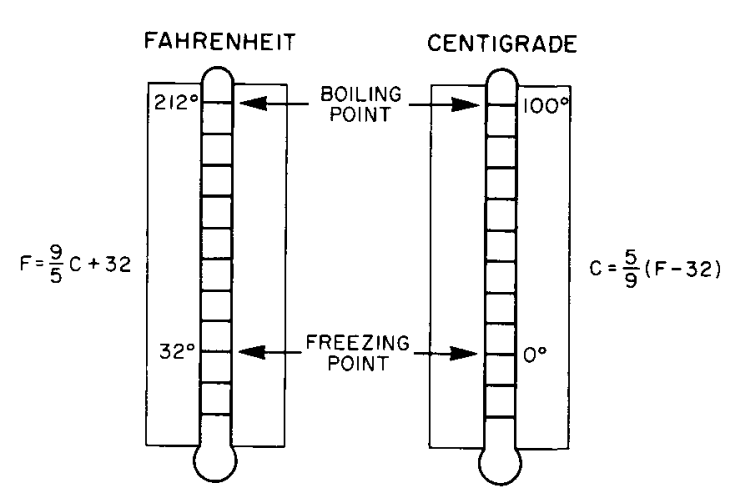

Fig. 5.0. Comparison of Fahrenheit and Centigrade Temperature Scales
Centigrade a difference of 100 degrees $\left(100^{\circ}-0^{\circ}\right)$. Thus, one Centigrade degree is equal to 1.8 Fahrenheit degrees, as long as the relationship involves just the size of the degrees on the two scales. To convert readings from Centigrade to Fahrenheit or vice versa, it must also be remembered that the two scales begin at different reference points. On the Centigrade scale, the freezing point of water is zero degrees, but on the Fahrenheit scale, the freezing point is 32 degrees above

zero. It should be obvious then that in order to obtain a relationship between the two scales related to the size of degrees, it is necessary to subtract or add 32 degrees to obtain the point where the respective scales begin.

Keeping these facts in mind, it is possible to write the following equations. To convert Fahrenheit to Centigrade, the formula

$$
C=(F-32) / 1.8
$$

is used, and conversely to convert Centigrade to Fahrenheit another form of the same equation,

$$
F=1.8 \mathrm{C}+32,
$$

may be used. The general formulae usually applied for temperature conversion are as follows:

$$
\begin{aligned}
& \text { Centigrade to Fahrenheit: } F=\left(C^{\circ} \times 9 / 5\right)+32^{\circ} ; \\
& \text { Fahrenheit to Centigrade: } C=\left(F^{\circ}-32^{\circ}\right) \times 5 / 9
\end{aligned}
$$

By use of one of these formulae, a reactor water temperature of $43^{\circ} \mathrm{C}$ can be converted to the Fahrenheit scale as follows:

$$
\text { Use Formula } F=\left(C^{\circ} \times 9 / 5\right)+32^{\circ}
$$

Therefore,

$$
\begin{aligned}
F & =\left(43^{\circ} \times 9 / 5\right)+32^{\circ} \\
& =77.4^{\circ}+32^{\circ} \\
& =109.4^{\circ}
\end{aligned}
$$




\subsection{Temperature-measuring Devices}

In the measurement of reactor temperatures, it is not feasible to use a conventional mercury or alcohol thermometer. Many points where such measurements must be taken are inaccessible, and an instrument must be capable of being read at a distance from the actual measuring point. In addition, it is advantageous if many widespread measuring points may be indicated centrally in an area such as the control room. If this can be accomplished, the reactor operator has an immediately accessible observation point from which to watch certain reactor characteristics. Two devices, the thermocouple and the resistance thermometer, adapt themselves readily to a remotely indicating system of this type. Both of these devices are used in Argonne reactors.

The first temperature-sensing device to be discussed is the thermocouple. A thermocouple is a pair of dissimilar, electrically conducting materials, joined together at one end. When two different metals are joined together at one end and the junction point is heated, a certain voltage will be developed between the free ends of the metals. This voltage is directly proportional to the temperature difference between the junction and the free ends, and falls in the millivolt range. If the free ends are then attached to a millivolt meter, the voltage may be read and converted to desired temperature units.

Many different types of metals will function in a thermocouple device, but certain metals do a better job than others. Consider the requirements in a good thermocouple scheme. First, the relationship between voltage output and temperature should be fairly linear. Metals providing a voltage change of about 0.1 millivolt per degree of temperature change are desirable for thermocouple use. The voltage changes must be reproducible over the anticipated operating range. Also, the voltages that the thermocouple develops must have a value sufficiently great to allow detection with standard measuring instruments. Finally, thermocouples are normally used in inaccessible points where high temperatures, rapid temperature changes, radiation damage, and corrosive effects are encountered. This means that the device must be capable of withstanding these effects over a reasonable operating lifetime.

No specific set of metals has been found that will function ideally under all conditions, but several different types are in common use. The operating conditions will determine the choice of a particular set of metals. Some common types of thermocouple wires are:

1. Copper-Constantan

2. Iron-Constantan

3. Chromel-Alumel

4. Chrome1-Constantan. 
The word thermocouple refers to the two specific metals and their junction, but this term is often used to describe the entire thermocouple assembly (see Figure 5.1). This assembly consists of the two appropriate metallic wires ( $A$ and $B$ ) surrounded by an

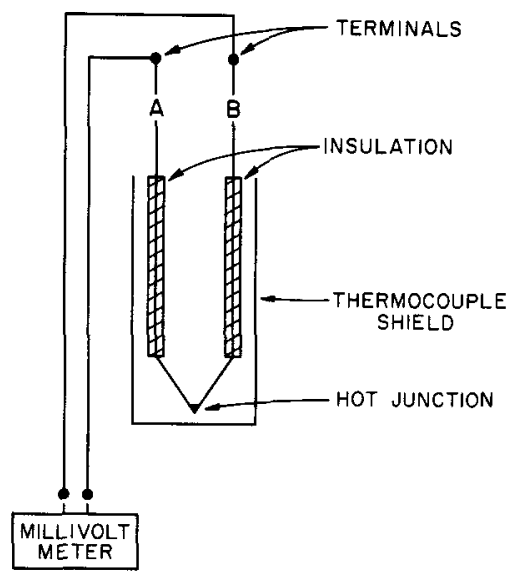

Fig. 5.1 .

Typical Thermocouple Assembly electrical insulating material. The entire unit is enclosed in a protective sheath which serves to minimize damage caused by heating and corrosion. At the upper end of the thermocouple assembly, terminal endings are attached to provide a means of connection to the measuring instrument.

As previously stated, it is desirable to provide for a centrally located point from which to receive various readings. As a rule, the thermocouple is not long enough to allow a direct connection to the measuring instrument. To make the circuit complete, extension wires composed of the same metals as the thermocouple wires are used. If extensions made of other metals were used, additional junctions would be created, and it would be very difficult to obtain an accurate temperature reading. An increase in the length of the thermocouple extension wires causes an increase in resistance. To compensate for this resistance, a measuring instrument called a potentiometer is often used. This device measures the voltage produced without requiring a flow of current. This instrument allows considerable flexibility in temperature measurement. It is possible to add any length of extension wire required and to locate all of the temperature-recording instruments at one place.

The thermocouple itself has two junctions. The first is the "hot junction." This junction is the point at which the thermocouple wires are joined and is subjected to the actual temperature that is to be measured. The other junction is a reference or "cold junction." This is the point at which the open ends of the thermocouple connect to the measuring instrument. Recall that the metals in a thermocouple unit produce a voltage, and that the voltage is proportional to the temperature difference between the junction and the open ends. Since this is true, it is important either to keep the reference junction at a constant temperature or to insure that the measuring instrument automatically changes as its temperature increases or decreases. If the reference junction fluctuates, temperature readings will not be accurate. It is more practical to provide for automatic compensation in the recording device than to be constantly concerned with maintaining an even temperature at the reference junction. To accomplish this, a copper coil is installed in the measuring instrument. This coil is designed to change in resistance as the temperature changes and to compensate for any temperature change in the reference junction. 
The second temperature-measuring device to be considered is the resistance thermometer (see Figure 5.2). In physical appearance, this device somewhat resembles the thermocouple. Its operation is based on

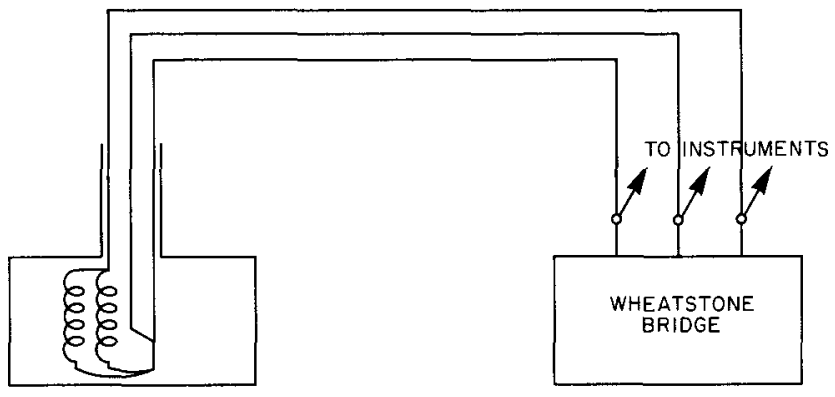

Fig. 5.2. Resistance Thermometer the principle that, when a metal is heated, its electrical resistance is increased. In the discussion on thermocouples, we were concerned with eliminating resistance from our measurement. The resistance thermometer uses the change of resistance to provide an accurate temperature reading. The device consists of a detecting element and a measuring instrument. The detecting element is called a resistance thermometer bulb. The bulb contains one coil of pure nickel wire and a second coil of manganin wire. The manganin coil is used to adjust resistance, and this feature makes all resistance thermometer bulbs identical and completely interchangeable. The nickel coil changes in resistance as temperature increases or decreases, and the change in temperature is recorded on a measuring instrument called the Wheatstone bridge. This instrument will measure the change of resistance in the nickel coil and indicate the change in terms of temperature. To compensate for changes of resistance in the extension wire, an additional conductor is usually added between the resistance thermometer bulb and the Wheatstone bridge. This wire compensates or balances out the resistance of the other two wires and provides for a more accurate reading. To prevent damage caused by corrosion and heat, the entire detection unit is enclosed in a protective sheath.

In measuring temperatures for reactor work, the difference between two temperatures, rather than the individual temperatures often becomes important. This temperature difference is called the delta $\mathrm{T}$ (and denoted as $\Delta \mathrm{T}$ ). In computing a power level, the temperature difference between two points in the primary circulating system is measured and multiplied by a precalculated factor to determine reactor power. Delta $\mathrm{T}^{\prime} \mathrm{s}$ are also important in informing the reactor operator as to the manner in which a particular system is functioning. To illustrate this point, briefly consider the catalyst or recombination chamber which has previously been discussed. In the catalyst chamber, elements formed by dissociation are recombined. A chemical reaction such as this produces a certain amount of energy in the form of heat. By measuring a delta $T$ across the catalyst chamber, it becomes possible to observe the efficiency of the chamber. This principle may be applied to many of the reactor systems and is another method of giving reactor personnel a wide scope of control. If thermocouples are used for measuring $\Delta T$, they need only be connected in series, as long as proper polarity is observed. When the resistance thermometer is used, $\Delta \mathrm{T}$ is measured by placing a second resistance thermometer bulb in another leg of the Wheatstone bridge circuit. 


\subsection{Necessity of Pressure Measurement}

Pressure is a force applied to a unit area. In a reactor, many different forces, both nuclear and physical, are at work. It is necessary to measure the pressures of gases and liquids. The reactor is essentially a closed system and therefore subject to internal pressure changes. Liquids are forced, under pressure, throughout reactor systems, and it is important that these pressures be observed and recorded. Helium pressures, air pressures, and others are also measured. As do temperatures, pressures keep the reactor operator informed as to the status of the various reactor systems. They provide a method of observing many internal changes, and also may serve to operate safety devices should extreme conditions arise.

\subsection{Pressure Scales and Units}

In measuring pressure, two different scales are in general use. These are the gage system and the absolute system. The only difference between the two systems is the reference point for zero pressure. In the absolute system, zero pressure is a complete vacuum, whereas in the gage system zero pressure is equal to the pressure of the earth's atmosphere. The absolute system of measurement is usually employed in scientific work, particularly when a high degree of accuracy is required.

Just as there are several types of instruments used for measuring temperature, so there are many instruments for measuring pressure. Since there are several units of force and several units of area, a variety of units of pressure have evolved. One common unit of pressure is "pounds per square inch." The various units arise because, in scientific work, it is convenient and accurate to measure pressure in terms of the height of a column of fluid which can be supported by a given pressure. One of the simplest instruments used is a barometer. A barometer may be constructed by filling a glass tube, about $80 \mathrm{~cm}$ long (and closed at one end), with mercury, and then inverting it and thrusting the open end into a well of mercury. The mercury column falls until its weight equals the weight of a column of air of cross section equal to that of the mercury column and extending from the surface of the mercury in the well to the top of the atmosphere. As the weight of the air changes, owing to changes in the atmospheric pressure, the level of the mercury in the tube moves up or down. The height of the mercury column, read from a meter stick placed alongside, thus serves as a measure of the atmospheric pressure.

The average barometer reading at sea level is 760 millimeters ( $\mathrm{mm}$ ) of mercury, a pressure known as the standard atmosphere. It is equivalent to 14.7 pounds per square inch (psi) or 1033 grams per square centimeter $\left(\mathrm{g} / \mathrm{cm}^{2}\right)$. In other words, on the average, a column of air one square inch in cross section and reaching from sea level to the top of the atmosphere, 
weighs $14.7 \mathrm{lb}$, and a column of mercury one square inch in cross section and $760 \mathrm{~mm}$ high weighs the same amount. The barometer measurement gives us a basis for converting other units of pressure measurement. Some of the other units and their conversion factors to psi are as follows:

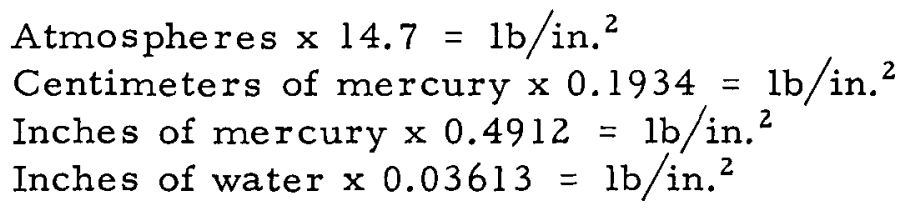

\subsection{Pressure-measuring Devices}

The manometer (see Figure 5.3) is one of the simplest pressuremeasuring devices in use. Basically, this device is usually a U-shaped glass tube that is partially filled with mercury, water, or a suitable material. If a pressure is applied to one side of the column, the level in
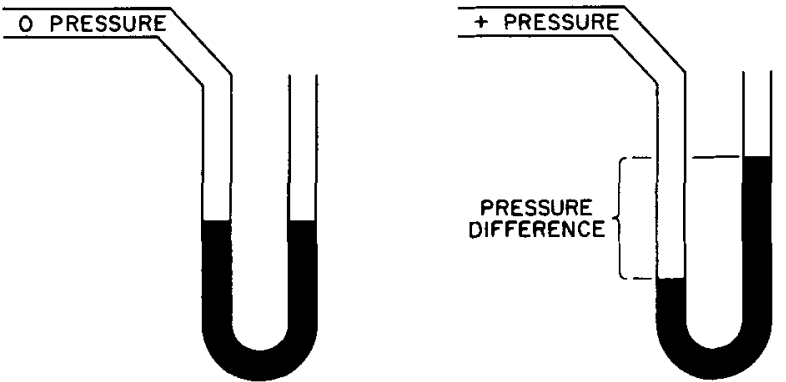

Fig. 5.3. Manometers that leg drops, and rises on the opposite side. The difference between these two levels is directly proportional to the applied pres sure. Considering the applied pressure as difference in the height, the device tells us that a given pressure is capable of supporting a column $h$ inches, centimeters, or other units of height. The column may then be calibrated to read in terms of the desired pressure units. Such a pressure will by necessity be read in the gage system, as one leg of the $\mathrm{U}$ tube is subjected to the atmospheric pressure. To provide for readings in the absolute system, it would be necessary to close one end of the tube, and seal it off from the atmosphere. This closed end must be completely evacuated or the pressure of the trapped air must be taken into consideration in the determination of the applied pressure. The pressure itself may be computed by multiplying the density of the liquid by the difference in height.

Another method of measuring pressure employs the use of a diaphragm mechanism (see Figure 5.4). This unit consists of a sealed box or case, pressurized positively, and containing a thin piece of corrugated stock, made from a material such as brass or stainless steel. The corrugated stock forms the actual diaphragm, and, when attached to this pressurized system, the diaphragm will bulge either inward or outward, as the pressure varies with respect to atmospheric pressure. This unit is capable of recording either positive or negative pressures. The bulging of the diaphragm may be related to a pressure reading by installing appropriate mechanical 
linkage between the diaphragm and a pointer and scale. As a force is applied, the diaphragm moves and the motion is translated to the pointer, which moves across a scale calibrated to read in terms of pressure.

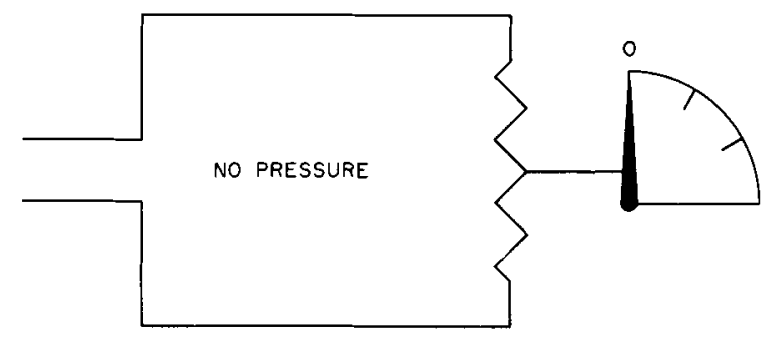

Diaphragm under Atmospheric Pressure

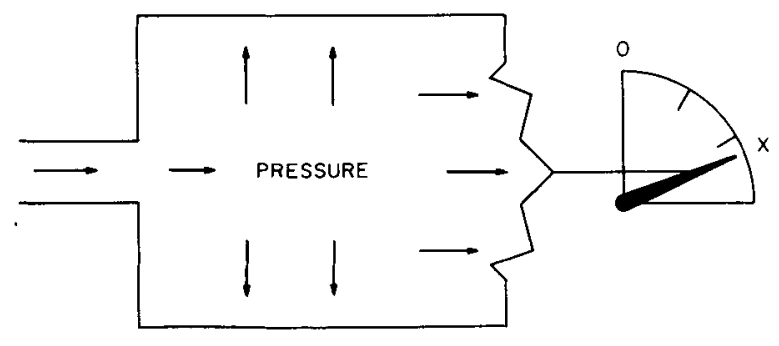

Diaphragm under " $\mathrm{X}$ " Pressure

(A)

(B)

Fig. 5.4A\&B. Diaphragm Pressure Gage

Still another pressure-measuring device to be considered is the bellows (see Figure 5.5). This unit operates in much the same manner as the diaphragm. The device is enclosed in a sealed case, and a spring mechanism is used to control the amount of expansion or contraction with changes in pressure. The spring provides a greater flexibility in the range of pressures that may be measured, as it may be adjusted to make the desired range either high or low. As either a positive or negative pressure is applied, the bellows move in or out, and the movement is translated to a mechanical device, which moves a pointer across a calibrated scale. The bellows has a greater mechanical advantage of motion than does the diaphragm. Pressures are normally read in the gage system, but may also be measured in the absolute system, by using a second bellows in series, from which all air has been evacuated.

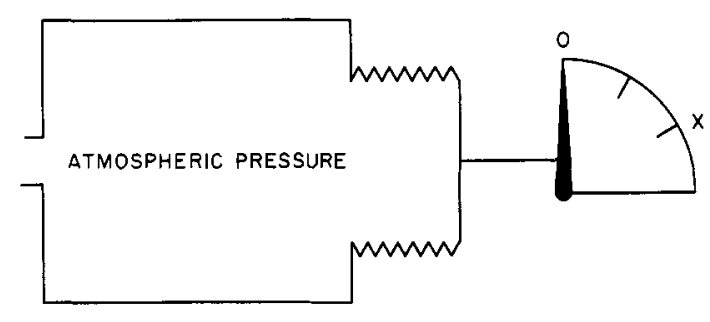

(A)

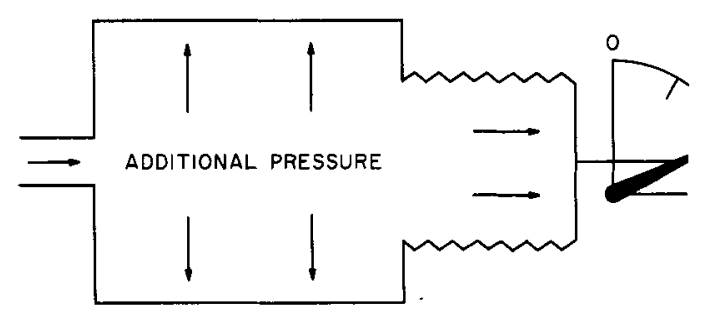

(B)

Fig. 5.5A\&B. Bellows Pressure Gages

The final pressure-measuring device that will be discussed is called the Bourdon tube (see Figure 5.6). Basically, this device consists of a spiral tube, normally elliptical in shape, and appropriate mechanical equipment. The tube is closed at one end and then curved into a spiral form. 
When a pressure is applied to the open end of the Bourdon tube, the spiral shape tends to unwind. A spring mechanism may be attached to a pointer, which will translate the unwinding of the tube to a scale, which is calibrated
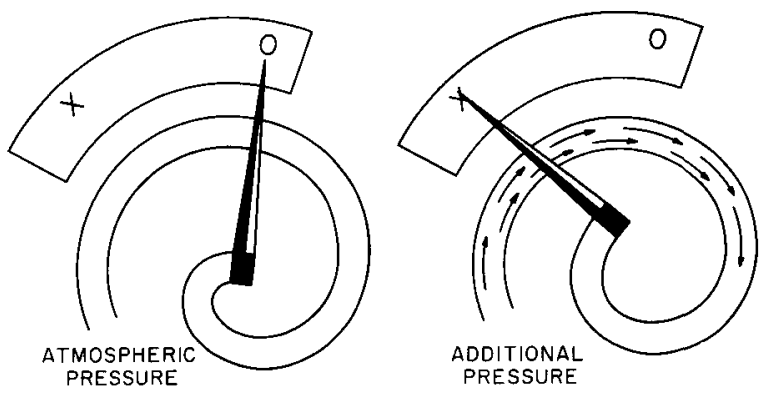

Fig. 5.6. Bourdon Tube to read in terms of pressure. The pressure is read in the gage system, and the Bourdon tube may be used to measure pressures both above and below atmospheric.

As was true of temperature measurement, the differential pres sure can provide certain important information on reactor behaviour. Three of the devices just discussed, namely, the diaphragm, manometer, and bellows, adapt readily to this type of differential measurement. In the case of the manometer, the two pressures to be measured are connected to the two ends of the $U$ tube. The difference in height of the two legs then becomes an indication of pressure differential.

In considering the diaphragm and bellows (see Figures 5.7 and 5.8), the situation is somewhat different. If the diaphragm is used, the two pressures are applied to the opposite ends of the diaphragm. The same arrangement will work with the bellows, but generally a separate bellows is used for each of the two pressures to be measured. These devices are not always connected directly to a scale, but rather signals are transmitted to an electronic device which has the ability to translate the various signals into the desired units. This device is sometimes a differential transformer. The unit consists of two coils of wire and a movable slug, which is attached to the actual measuring device. As the diaphragm or bellows moves, the slug moves up or down the center of the two coils, and the coupling is varied. If an AC signal is applied to one coil, the other coil will yield an AC signal. This signal is proportional to the relative position of the metallic slug. This signal is measured and related to the physical position of the diaphragm or bellows. To measure differential pressures with this type of device, a diaphragm with two chambers might be used. Of course, the ordinary pressure

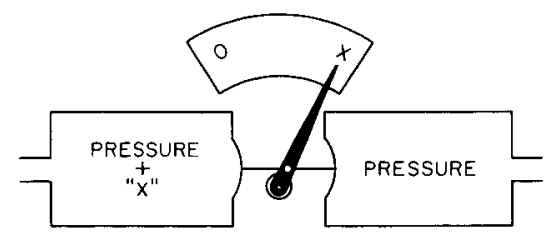

Fig. 5.7. Differential Pressure Measurement - Diaphragm

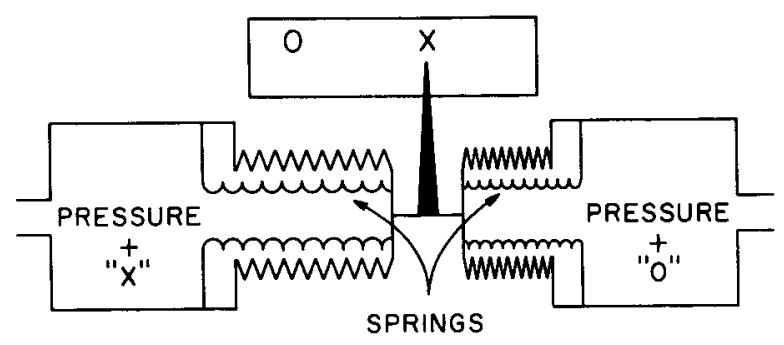

Fig. 5.8. Differential Pressure Measurement - Bellows 
gage may also use an electrical transmission for measurement. The position of the diaphragm is related to the pressure difference between the two chambers. A mechanical means is used to send the actual movement to the transformer, and the signal is transmitted.

To use a bellows arrangement, two separate bellows are employed. The applied force will push the bellows in either of the two directions. The center connector will move back and forth, due to differential pressure, and this movement may be transferred to the differential transformer. It should be apparent that in reactor work we are readily able to obtain differential pressures and temperatures as well as the absolute temperatures and pressures themselves.

\subsection{Liquid Flow and Units}

Another important variable that is measured in and around a reactor is flow. This discussion will be limited to descriptions of liquid flow and the various devices necessary in flow measurement. Liquid flows in primary, secondary, and other support systems are commonly measured in reactor work. The flow rate in the primary system is especially important in computation of the reactor power level. In discussing flow measurement, it becomes necessary to differentiate between the measurement of flow rate and flow volume. Flow volume is simply a measurement of the total volume in gallons or other convenient units. Ordinary water meters yield this type of measurement, and in certain reactor measurements this type of device will suffice. Generally speaking, however, a flow rate or a volume passing a given point per unit time is measured. The units of flow are measured in terms of a given mass or volume per unit time. Reactor rate of flow instruments are almost always calibrated in units of the number of gallons per minute.

\subsection{Liquid Flow-measuring Devices}

Such measuring devices as the orifice, nozzle, and venturi operate under the same basic principle. This principle states that a pressure drop caused by a restriction in flow is proportional to the actual flow. The relationship may be expressed by the formula

$$
Q=K \sqrt{H} \text {, }
$$

where $Q$ is the flow rate, $K$ a constant depending upon characteristics of the installation (including the specific gravity of the liquid being measured), and $H$ is the pressure drop across the artificial restriction.

The first measuring device to be discussed which uses this principle is the orifice (see Figure 5.9). The orifice is simply a thin stainless steel plate. The plate is designed with a predetermined opening for a particular 
size piping scheme. Information is provided by the manufacturer as to the pressure drop caused by the restriction, and this information is used in calibrating the electronic recording instruments. To measure the flow rate

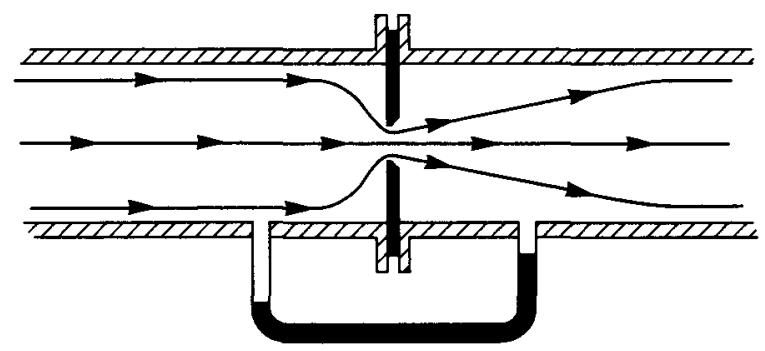

Fig. 5.9. Sharp-edged Orifice Flowmeter in a particular system, the orifice plate is installed in the piping between two flanges, and the drop in pressure across the orifice may be related to flow by the formula previously mentioned. Normally, the opening in the orifice is circular and located in the center of the plate. This arrangement is not mandatory, and, indeed, orifices are made in square, triangular, and elliptical shapes. The orifice may be changed in shape and location, as requirements of a particular reactor system demand. If the liquid to be measured contains a great deal of particulate matter, it may be necessary to use a segmental orifice, almost flush with the bottom of the pipe to prevent clogging of the system.

A second device, called the nozzle, is quite similar to the orifice plate. In reality, it is an orifice plate with flared or rounded approach sections. This device behaves in a manner similar to the orifice plate, and the flow rate is calculated by the same formula; $Q=K \sqrt{\mathrm{H}}$, but different constants $K$ must be used. The device is somewhat more efficient than the orifice and is particularly advantageous in reactor systems in which the liquid medium contains solids in suspension. In the orifice plate, solids can easily dam up behind the plate, producing a clogging of the system, whereas the rounded or tapered sections of the nozzle provide greater freedom of motion. The requirements of the system will determine the specific measuring device used.

The best device of this type that we will consider is the venturi (see Figure 5.10). This unit is by far the most efficient of the three. Its main disadvantage is one of cost and size. The unit itself is bulkier than the orifice and nozzle devices, and may present installation difficulties. The

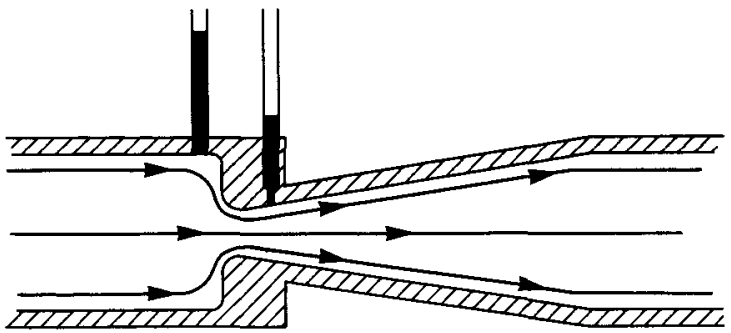

Fig. 5.10. Venturi Meter venturi consists of two tapered sections of piping placed together in a manner which results in a continuous tapered restriction. The pressure drop is measured between the upstream portion of flow and the throat of the venturi.

It is relatively easy to obtain a pressure drop across a restriction 
and to use this pressure difference in computation of flow rate. It would not be convenient or practical to use a formula each time knowledge of a given flow becomes necessary. To accomplish this, the differential pressure is usually measured by an instrument such as the diaphragm. Generally, the diaphragm is connected to the recording instrument by means of an electrical or pneumatic device. To calibrate the instruments, it is necessary to think in terms of the formula for calculating flow rate and to extract the square root of the differential pressure. This may be done either electrically or mechanically, or by simply calibrating the scale with squareroot graduations. Again, requirements of the system dictate the method to be used.

The rotameter (see Figure 5.11) is still another important flowmeasuring device. These units may vary somewhat in their general appearance, but are all basically alike in method of operation. The rotameter is composed of a float of some given weight. The float is free to move through-

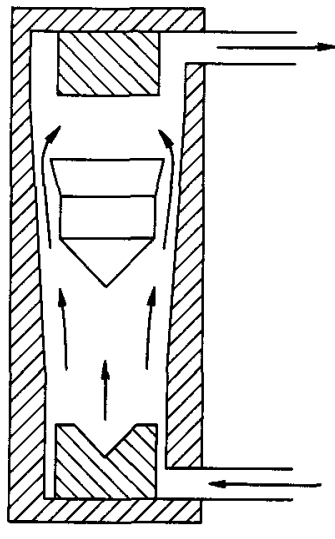

Fig. 5.11.

Rotameter Flow

Measurement out a vertical plane within a metal tube or tapered glass. The smaller section of the tapered tube is at the lower end, and liquid is free to flow in and through the unit. As the liquid to be measured enters the rotameter, it exerts a certain pressure upon the float. This pressure causes the float to rise, and, as it rises the annular area between the float and the tapered tube increases. (Observe that the direction of flow is upward.) When the area increases, the pressure drop created by the restriction formed by the float, and the tube of course, decreases. At some point, the weight of the float will be exactly balanced by the differential pressure, and the motion of the float will be arrested. The rate of flow will be indicated by the position of the float. If the tapered unit is made of glass, this section may be calibrated in terms of flow rate and read directly from the position of the float. When metallic sections are used, mechanical or electrical devices become necessary in determination of float location. The calibration of the instrument depends upon the taper of the tube section, weight and shape of the float mechanism, and the specific gravity of the liquid to be measured.

The last flow-measuring device to be considered in this discussion is the turbine (see Figure 5.12). These devices are based upon the principle that a fixed device installed with a provision for rotational movement within a flow stream will move at an angular velocity proportional to the flow rate. The turbine, then, is a propeller-type device installed at some point in the piping system where flow measurements are required. 


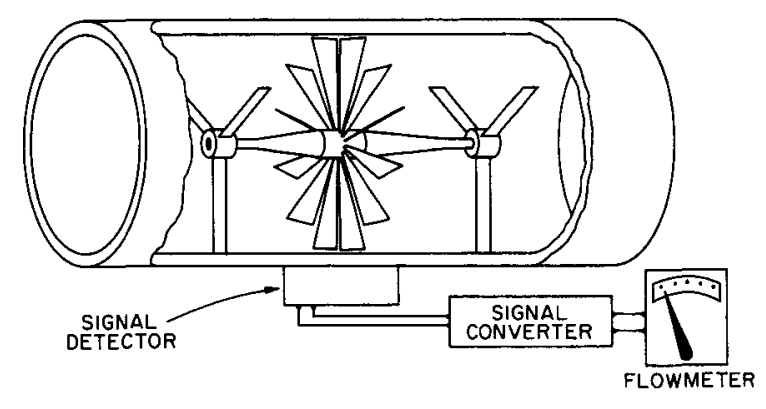

Fig. 5.12. Turbine-type Flowmeter

As the liquid flows through the pipe, a force is exerted upon the turbine blades. This force causes the blades to rotate, and the angular velocity of the blades is proportional to the actual flow rate of the liquid. A unit of this kind, well constructed and accurately calibrated, is capable of measuring flow rates within one per cent or even better. This high degree of accuracy usually justifies the higher cost of this type of measuring device.

To provide a means of measuring the rotational velocity of the propellor blades, magnets are attached to one or more of the vanes. As the magnet rotates with the vane, it passes a stationary pick up coil. Each time the magnet passes this coil, a small voltage is induced. An electronic device capable of counting these voltage pulses generates an output voltage which is proportional to the actual speed of the turbine. An appropriate voltagemeasuring device calibrated in units of flow rate may then be used. As previously stated, these units will normally be in gallons per minute.

\subsection{Liquid Levels and Units}

Throughout the various reactor systems, liquids are used. The reactor vessel contains a suitable moderator in the form of a liquid, and various liquids are stored in expansion tanks, dump tanks, and the like. The reactor operator must be aware of liquid-level status in these areas, and it is necessary to develop a convenient method of level measurement in these areas. If storage tanks were easily accessible to the operating group, a simple measuring device would be of the familiar dip-stick or ruler type. This method, however, is not feasible in reactor work. The normal method in measuring liquid level is in terms of the height of the liquid.

\subsection{Devices for Measurement of Liquid Level}

One of the simplest type of level gages currently in use is the sight glass (see Figure 5.13). This device resembles a manometer and in reality is one leg with the other leg of manometer being the tank itself. The main qualification for this type of device is that the level in the stand pipe must be the same level as that of the tank. The level of the liquid present in the sight gage varies in the same manner as the level in the tank. A suitable scale may be etched on the glass itself, either to measure the liquid level in terms of height or by calculation of the amount of liquid in gallons. 


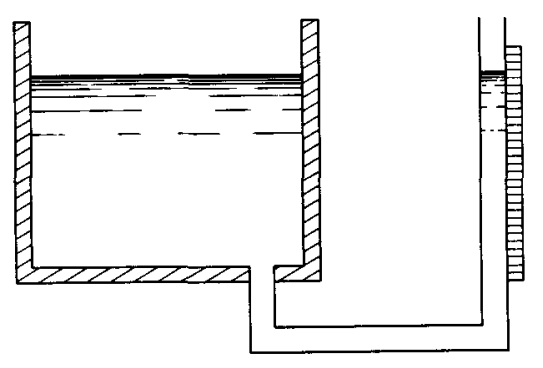

Fig. 5.13.

Liquid-level Measurement by Sight Glass
A second simple level indicator to be discussed is the float (see Figure 5.14). These devices are used in reactor tower basins to maintain preset water levels and for other applications. A mechanical device connects the float unit to the core section of a differential transformer. The float, generally constructed of aluminum, is joined to the transformer with a movable rod. As the liquid level changes, the rod moves either up or down within the trans former and changes its inductance. This inductance change is proportional to the amount of core present within the coils, and this

amount is proportional to the level of the measured liquid.

Although the sight glass and float are the most direct means of measuring liquid level, pressures may also be used to determine liquid levels. Any type of pressure-meas uring device may be positioned in a lower portion of the vessel containing a liquid to be measured and recalibrated in terms of height instead of pressure. Caution must be exercised, however, if the measuring device is located at a point above the bottom of the vessel. Generally, a differential pressure device will be employed because in most cases the atmosphere above the level of

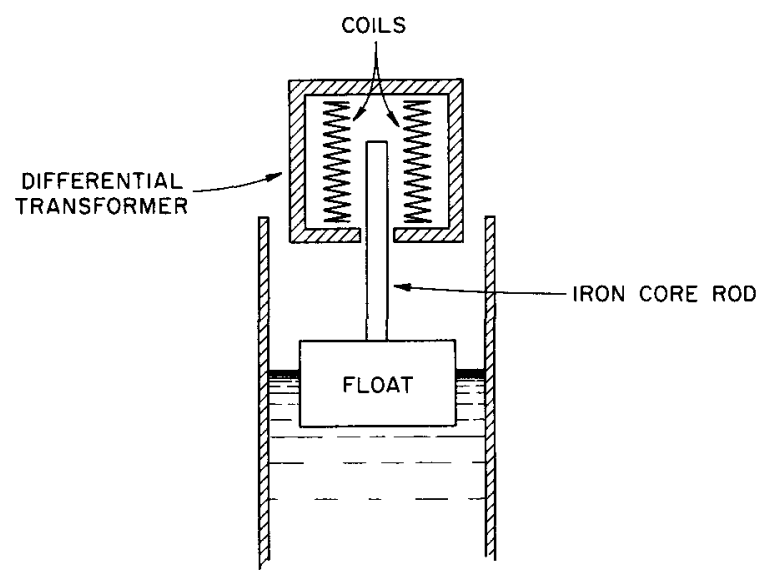

Fig. 5.14. Liquid-level Measurement by Float

the liquid in the vessel will be slightly pressurized. To relay the position and movement of a diaphragm or bellows in this type of arrangement, the movement is translated into an electrical signal by either a differential transformer or a strain gage. Basically, the strain gage is a device which changes in electrical resistance as it is deformed. The strain gage is attached to the diaphragm or bellows, and as these devices become subjected to pressures, also becomes distorted. The distortion alters the electrical resistance of the strain gage. Recording instructions may then be calibrated to read level in terms of electrical resistance. Whenever a liquid level is measured by means of a pressure-measuring device, corrections must be made to compensate for any fluctuation in the density of the liquid due to changes in temperature, as the actual measurement involves the weight of the liquid. If the density of the liquid is changed, the indicated height will also change. 


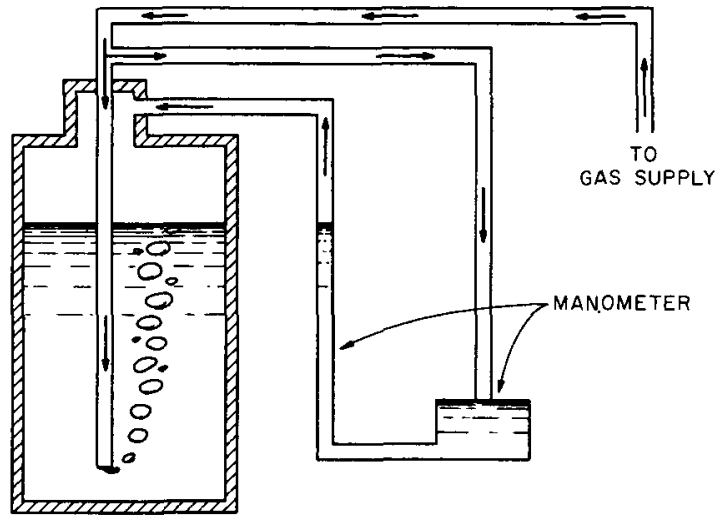

Fig. 5.15. Bubbler Device
One of the more complex level-indicating devices consists of a tube or probe immersed in the liquid whose level is to be measured (see Figure 5.15). The inlet of the probe is pressurized, which causes a bubbling of gas up and through the liquid. The pressures required to cause a constant rate of gas flow are proportional to the height of the liquid above the bottom of the tube. A device is used to vary the gas pressure and keep the flow constant.

Of course, the higher the level, the higher will be the pressure to cause bubbling of gas. This pressure may be measured with appropriate pressure instruments, calibrated in terms of the liquid level.

The last level-measuring gage to be considered operates upon the principle of the electrical capacitance effect. The capacitance of a suitable condenser probe will vary with the level of the liquid being measured, and the measurement of capacitance will yield a direct level reading. To use this type of instrument, generally, two electrodes are placed in the liquid to be measured. These electrodes or conductors form the plates of a capacitor and the liquid acts as a dielectric. The capacity measured across these conductors will vary with the height of the liquid. Essentially, the ratio of air to liquid is changed, and the dielectric constant is measured. If the temperature of the liquid changes, the dielectric constant will be changed, and correction factors will be needed. As only capacity is being measured, the probes may be insulated with materials such as Teflon to prevent corrosive effects on the probe units. The advantage of this type of measurement is one of simplicity. The device has no moving parts and may be readily located within a pressurized system. In addition, changes in atmospheric pressure will not affect instrument accuracy. 


\section{CHAPTER VI}

\section{NUCLEAR INSTRUMENTS}

\subsection{Necessity of Nuclear Detectors and Ranges}

Some of the more basic principles of nuclear fission, reactor design, support systems, shielding, and temperature and pressure measurements have been discussed. Nuclear detectors or instruments are generally considered as instruments that will detect nuclear particles or the emissions from various nuclei within the reactor. Nuclear instruments measure neutrons and gamma rays. It may generally be assumed that the power level of a reactor is proportional to the number of neutrons in its core. Of course, operating conditions arise in which the relationship between numbers of neutrons and power is much more complex, but for the purposes of this book the assumption of direct proportionality will be taken as correct.

A problem exists in measuring a given fixed fraction of the reactor neutrons with conventional nuclear detectors, and then relating the output to reactor power. To start with, it may be as sumed that the neutron-flux distribution in a reactor is not constant, but will vary with time and position in the core. To obtain a power level directly, it becomes necessary to measure neutron flux continuously. To accomplish this measurement, a large number of detectors may be placed uniformly throughout the core, and an average taken of their individual output readings; secondly, a detector might be placed far enough away from the core so that the core may be considered as a neutron point source, and then local neutron-density variations in the core will become insignificant. As in other reactor work, both methods require certain compromises to be at all applicable.

The fact that detecting instruments are usually located at some distance from the reactor places limitations upon instrument sensitivity and range. Remember that a nuclear reactor is never really shut off, but just shut down. In a conventional plant whose normal power or heat output might be $5,000 \mathrm{kw}$, if the output is shut down to $1 \mathrm{kw}$, the plant would be considered essentially shut off and could be ignored. This is not true in nuclear operation for the case of a nuclear reactor operating at the same level. One kilowatt still corresponds to a great number of neutrons. Recall the unique neutron-multiplying characteristics of the medium at any level (see Sections 1.8 and 1.9).

As a reactor is capable of creating a multiplication factor greater than unity, the neutrons must be continuously monitored for safety reasons. The reactor operator needs a constant automatic method of monitoring internal reactor behavior. Neutrons, then, must be monitored at all times and at all levels. In the case of power reactors, requirements may call 
for instruments having a range of as much as 10 decades to provide complete coverage of the entire range from source strength to full operating level.

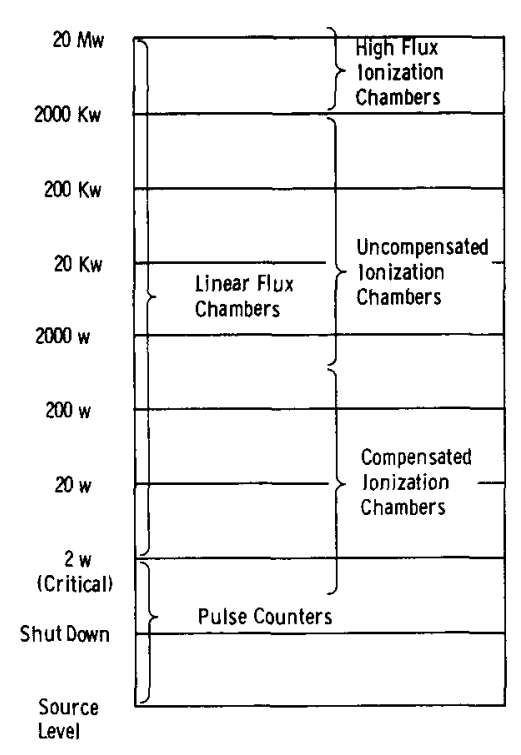

Fig. 6.0. Multiple Instrument System
Unfortunately, no one present-day instrument is capable of covering such a wide range. Detectors usually can operate effectively over only three, or at most four, decades. Consequently, to cover the wide requirements of a nuclear reactor, complete coverage must be obtained by using many instruments with overlapping ranges. Figure 6.0 indicates the multiple instrument system.

The entire range from source strength to operating level is divided into decades, and specific types of instruments are assigned for operation at all of the given levels. You will note that in this scheme the reactor becomes critical seven decades below the actual operating level. Below the critical range and at the shutdown level, counters are used. Above the critical level, by necessity, ionization chambers

are used. These chambers must be divided into two classes, according to sensitivity. High-flux ionization chambers cover the upper two and one-half decades of operation. Linear flux chambers cover all the inbetween ranges above critical.

\subsection{The Ionization Chamber - Uncompensated and Compensated}

The neutron-sensitive ionization chamber is normally used for measurement of neutron flux in the intermediate and power ranges. These are the instruments which are usually connected to the automatic control systems.

Because the neutron is an uncharged particle, recourse must be made to an intermediate reaction producing charged particles, which may be detected. Consider a charged-particle detector (see Figure 6.1). Basically, the detector consists of a cylinder closed at both ends. The cylinder is filled with some suitable gas such as argon, nitrogen, or methane. A wire is passed through an insulator on the axis of the cylinder and maintained with a negative voltage with respect to the cylinder.

A charged particle entering the chamber strikes the atoms composing the gas and in the process strips electrons from the se gas atoms. This process leaves the gas atom in a state of positive charge. Such an atom is said to be "ionized" and is often referred to as a positive ion. 


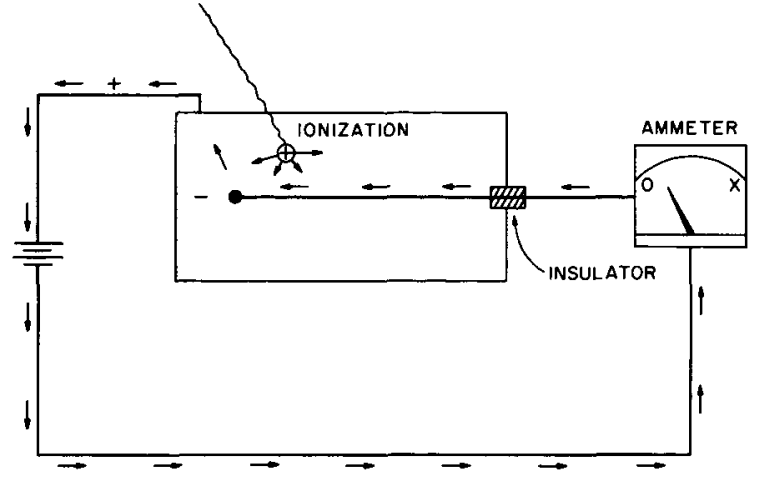

Fig. 6.1. Charged-particle Detector
As more charged particles enter the chamber, more ions are created. As a result, the chamber will contain a number of positively charged ions and also free, negatively charged electrons. As the wire through the cylinder is maintained with a negative voltage, the positive ions will be swept toward it. The negatively charged ions will be attracted to the positive charge maintained at the surface of the cylinder.

It should be apparent that with a sufficient number of charged particles, the center wire will tend to become positive, and the electrons will flow in an external circuit to neutralize the central wire.

A micromicroammeter may be installed in the external circuit to measure the flow of electrons. When a continuous flow is produced, this flow will be proportional to the flux of the charged particles entering the cylinder, and thus a measurement is provided of charged-particle flux.

We have stated that the neutron carries no electrical charge. Obviously, in measuring neutron flux for power level and control purposes, a method must be devised to cause the neutron to produce charged particles. The neutron ionization chamber will accomplish this. Basically, the ionization chamber consists of the charged-particle detection unit just discussed, but the system will not work unless there is an intermediate step in which neutrons produce charged particles. To accomplish this, the detector consists of chambers in which large surfaces may be coated with boron-10 and the ionization from the recoil alpha particles again collected as current. The actual nuclear reaction occurring is

$$
{ }_{5} \mathrm{~B}^{10}+{ }_{0} \mathrm{n}^{1} \rightarrow\left[{ }_{5} \mathrm{~B}^{11}\right] \rightarrow{ }_{3} \mathrm{Li}^{7}+{ }_{2} \mathrm{He}^{4}
$$

This reaction occurs in an ionization chamber when neutrons come in contact with boron-10, either as boron plated on the electrodes or as boron trifluoride $\left(\mathrm{BF}_{3}\right)$ in the chamber gas. The neutron comes within the sphere of physical influence of the boron atom and is absorbed by the atom, momentarily forming boron-11. The boron-11 atom is unstable, and breaks apart, forming lithium-7 and helium nuclei. You will recall that the helium nucleus is simply an alpha particle, which has a positive charge. The reaction may be written in shorthand form

$$
{ }_{5} \mathrm{~B}^{10}(\mathrm{n}, \alpha)_{3} \mathrm{Li}^{7}
$$

where $\mathrm{n}$ or neutron represents the bombarding particle and alpha the emitted particle, the reaction is often referred to as an " $n$, alpha reaction." 


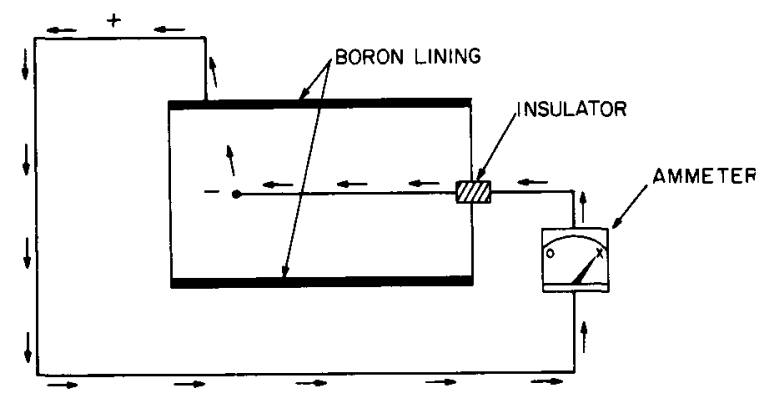

Fig. 6.2

Uncompensated Ionization Chamber
The uncompensated ionization chamber (see Figure 6.2), then, is simply a charged-particle detector based on an $\mathrm{n}$-alpha reaction for the measurement of neutron flux. The neutrons produce alphaparticles which produce a current via ionization of gas molecules. The current is proportional to neutron flux and may be measured by suitable instruments. The ionization chamber produces a DC current which the measuring device requires to function properly. At subcritical power levels the ionization chamber produces a pulsating current due to the low neutron intensity, and so it cannot be used in this region.

Gamma rays also have the ability to ionize atoms, and, unfortunately, an uncompensated ionization chamber detects gamma rays as well as neutrons. At shutdown levels, the fission products are a considerable source of gamma rays. At these levels, then, the ionization chamber still produces a current. If this current is not deleted from the current actually produced by incoming neutrons, changes of neutron flux during startup may be completely masked by the gamma rays. To eliminate faulty readings caused by the presence of gamma rays, a compensated ionization chamber (see Figure 6.3) is constructed so that gamma-ray-induced currents are automatically subtracted from total current readings. The resulting net current produced by the chamber will represent only neutron interaction.

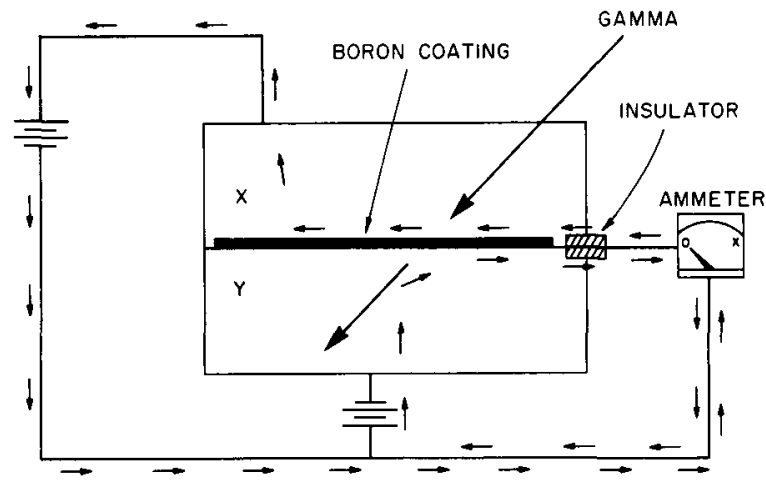

(A)

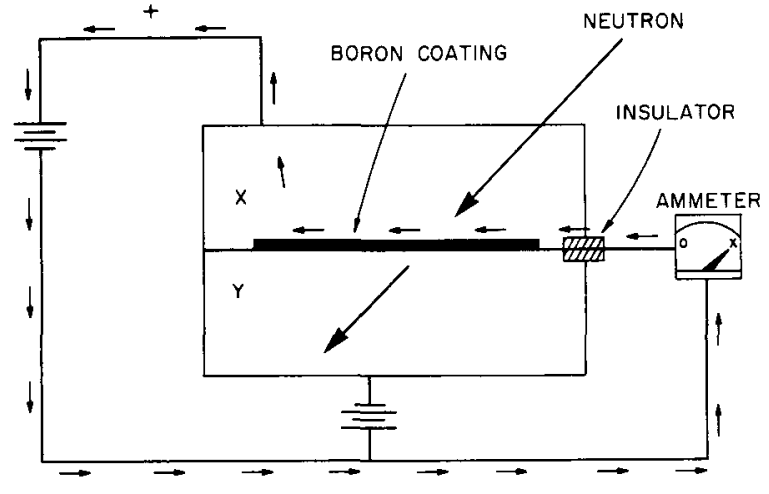

(B)

Fig. 6.3A\&B. Gamma-compensated Ionization Chamber

To accomplish this, two identical chambers are constructed (see Figure 6.3, $\mathrm{A}$ and $\mathrm{B}$ ). Only the upper chamber $(\mathrm{x})$ contains a boron coating. A positive charge is maintained on the upper plate and a negative charge on the lower plate. As the lower section contains no boron, any current 
produced will be entirely due to gamma rays (see Figure 6.3A). Any gamma-induced positive ions will be repelled by the positive charge in the upper chamber toward the common electrode. Conversely, any gammainduced electrons in the lower, negatively charged chamber will also be repelled towards the common electrode. This results in a current flow equal in magnitude, but of opposite charge, with a net result equal to zero at the collecting electrode.

Recall that the upper cylinder contains boron. As neutrons enter the chamber, the ${ }_{5} \mathrm{~B}^{10}(\mathrm{n}, \alpha){ }_{3} \mathrm{Li}^{7}$ reaction occurs, and additional positive charges are produced within the chamber (see Figure 6.3B). These charges result in further collection at the common electrode, and an external current will flow which may be measured as neutron-produced current.

Both compensated and uncompensated detection units are used in reactor work. In upper power regions, uncompensated chambers are used, as the neutron flux is of sufficient intensity that the gamma current is insignificant. The lower regions require the use of compensated chambers due to the intensity of gamma rays versus neutron intensity.

\subsection{The $\mathrm{BF}_{3}$ Proportional Counter}

We have stated that the ionization chamber produces a continuous current. In radiation fields not high enough to produce this current, counters must be used to detect individual neutrons present. The most sensitive type of pulse-counting instrument is the $\mathrm{BF}_{3}$ counter. The $\mathrm{BF}_{3}$ proportional counter, in common with most of the other neutron detectors, relies on the detection of ionized particles which are produced by the now familiar $\mathrm{B}^{10}(\mathrm{n}, \alpha) \mathrm{Li}^{7}$ reaction.

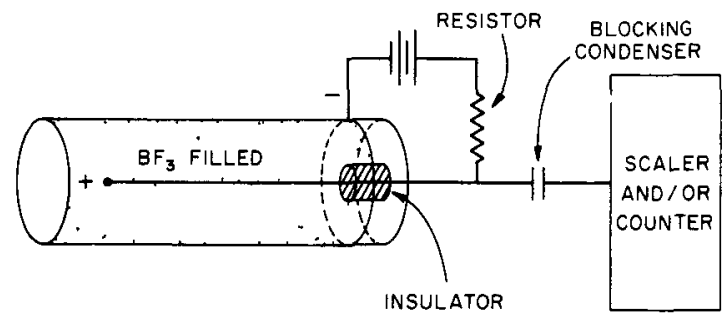

Fig. 6.4. $\mathrm{BF}_{3}$ Proportional Counter
Briefly, the counter (see Figure 6.4) consists of a metal cylinder with a wire of small diameter suspended coaxially within the cylinder. In a counter the wire is always positive and maintained at a high potential. This cylinder is usually filled with a gas containing boron trifluoride $\left(\mathrm{BF}_{3}\right)$. This gas is ionized by the alpha particle emitted in the reaction. The electrons formed from the primary ionization are accelerated towards the collecting electrode by the electric field in the counter. In doing so, the se electrons acquire sufficient energy to ionize other molecules. It should be apparent that the total number of electrons increases as the wire is approached. If we limit the process to one particle entering the chamber, one electron will be removed. This electron moves swiftly toward the electrode, and in so doing removes another electron, which in turn removes still other electrons in a cascading effect. This 
process is called "gas multiplication." When large numbers of electrons are collected at the electrode, the voltage of the wire will change abruptly. When all of the available ions have been collected, the counter returns to a quiescent state, until another ionizing particle enters the chamber. The rate at which these pulses appear upon a measuring instrument is a linear function of the neutron-flux level in which the counter is located.

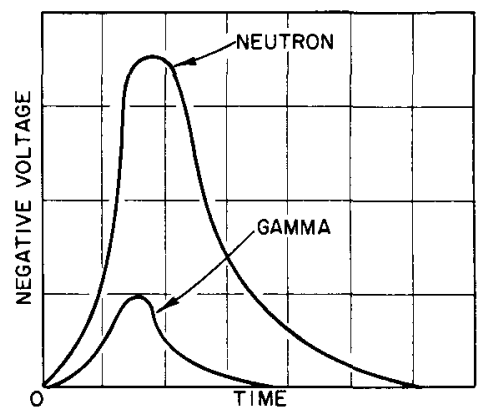

Fig. 6.5

A typical voltage pulse as it would appear upon the wire is depicted in Figure 6.5. This pulse may be coupled to an appropriate counting circuit, and in this manner individual particles may be counted. It is not necessary to compensate a counter of this type, as the difference between neutron and gamma pulses is readily distinguished. To facilitate gas multiplication, the $\mathrm{BF}_{3}$ counter operates with very high potentials. These instruments are always turned off as soon as they have fulfilled their startup functions. Due to the large gas multiplication in a pulse counter,

Neutron and Gamma Pulses the instrument may not be used for high flux measurement. They will be damaged if left turned on in a high flux.

All of the detection devices discussed in this chapter will also count gamma radiation. To accomplish this, a chamber is designed in which materials are chosen that will enhance sensitivity to gamma rays; the use of heavy gases within the chamber and of heavy metals for the electrodes will increase this sensitivity. The requirements for any reactor control instrument include high neutron sensitivity, gamma-radiation insensitivity, or, if gamma radiation is to be measured, the opposite effect, and construction to be such that neutron-induced radioactivity in the detector be held at a minimum.

\subsection{Detector Circuits}

We have discussed the currents produced by various nuclear detectors. Different circuits are necessary to measure the se currents. Generally, the current produced in an ionization chamber is passed through a resistor, which then produces a voltage across the resistor. The voltage produced is proportional to the current flowing from the chamber. The voltage may then be amplified to a level capable of operating recorders or trip circuits.

Consider the system illustrated in Figure 6.6. The collecting electrode is joined to a resistor which is connected to ground. A power supply, such as a battery, supplies the voltage for the circuit. Current flows through the electrode, which causes a voltage to be produced across 


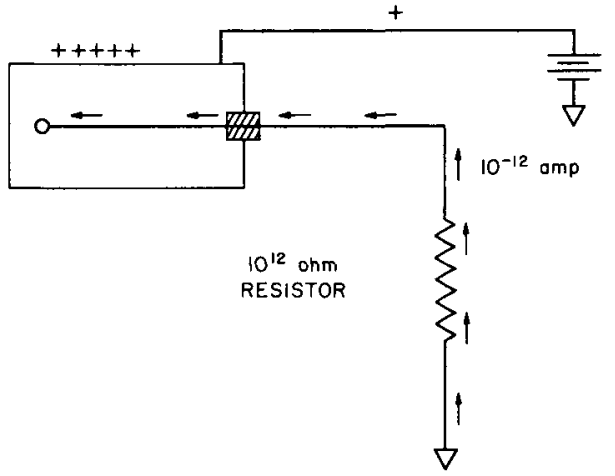

Fig. 6.6

Chamber-currentmeasuring Circuit the resistor. This voltage is measured. If a current of $10^{-12}$ amp is flowing from the electrode through a $10^{12}-\mathrm{ohm}$ resistor, the net measured result is one volt. This is the manner in which most current meas uring circuits operate. If it is necessary to change the detection range, the resistor is changed.

When pulse counters are used, scalers are normally placed in conjunction with the counter to record pulses. The scaler is a device which will record one pulse after a given number of pulses are put into it. For example, a one-decade scaler yields one pulse for every ten pulses put into the device. Most scalers used in nuclear work are of the three-decade type. This device is necessary to keep mechanical registers, which are only capable of counting a limited number of pulses per second, from jamming.

Another device which takes pulses and measures the rate at which they occur is the count rate meter. These instruments are efficient only at pulse rates greater than several per second and are not nearly as accurate as the scaler, which records individual pulses.

\subsection{Flux Channels}

Let us now consider some of the instruments necessary both to measure neutron flux within a reactor and to act in shutting the reactor down if the neutron intensity reaches a predetermined set point. The first instrument to be considered is called the high-flux channel. This type of channel is almost never used as an indicator for reactor power level, and, in general, the channel is used only in the upper two and one-half decades of the reactor range. The complete channel consists of an ionization chamber, of the uncompensated variety, which is connected to an amplifier. The amplifier takes the chamber current and produces a voltage proportional to the incoming current. It has been previously noted that this voltage will also be proportional to the neutron flux. The voltage may be used in limiting the reactor power level. The chamber-produced voltage is sent to a trigger or trip circuit. If the neutron-induced voltage exceeds some preset safety limit, the trip circuit will operate, allowing the safety or control-rod release mechanism to drop the safety rods into the reactor core, automatically providing a reactor shutdown. The highflux channels are usually multiple and failsafe. A failure of one component does not leave the reactor unprotected. 
Linear-flux channels are essentially the same as high-flux channels except that they cover a much wider operating range. These channels are used to provide the operator with a wide picture of the reactor power level. The channel consists of an ionization chamber which is connected to a multirange amplifier. The magnified signal goes to a recorder or a galvanometer. This type of instrument provides a very accurate readout within the calibrated range.

The last flux channel to be considered in this discussion is called a log-flux channel. Very basically, this type of instrument depicts reactor neutron flux on a logarithmic scale. In this manner, the operating range of neutron flux may be described on one single graphical picture without making changes in range. The fine details of reactor behavior in a given decade are lost, but the instrument does provide a general picture over the entire operating range. The ionization chamber in this channel produces a current $i$, which is fed into a logarithmic amplifier. The log amplifier in turn produces a voltage proportional to the logarithm of $i$, and this is displayed upon the desired recording instrument.

\subsection{The Period Channel}

Another circuit which is peculiar to reactor control is the period channel. This channel yields an output proportional to rate of change of neutron level. Period outputs are obtained by first starting with a signal proportional to power level. The logarithm of this signal is then obtained in a logarithmic amplifier, and finally the time derivative of the logarithmic signal is taken to provide an inverse period signal. The heart of this operation is the logarithmic amplifier, which also is used to compress the wide range required. Recall that the reactor period may be defined as the time required for a reactor to change in power by a factor of e, equal to 2.718 (see Section 1.10). It should be apparent that a period channel must in some manner measure either the period or something that is proportional to the period. Review for a moment the equation depicting reactor behavior. This equation states that

$$
\mathrm{P}=\mathrm{P}_{i} \mathrm{e}^{\mathrm{t} / \tau} \text {. }
$$

Upon taking natural logarithm,

$$
\ln \mathrm{P}=\ln \mathrm{P}_{\mathrm{i}}+\ln \mathrm{e}^{\mathrm{t} / \tau}
$$

therefore,

$$
\ln \mathrm{P}=\ln \mathrm{P}_{\mathrm{i}}+\mathrm{t} / \mathrm{T}
$$

We observe in equation $(6 c)$ that the reactor is changing in power level from the initial power $P_{i}$ to the power $P$. Therefore, the rate of change 
of $(\ln P)$ equals rate of change of $\left(\ln P_{i}\right)+$ rate of change of $(t / \tau)$. The initial power is some fixed value, and therefore has no rate of change, so rate of change of $\ln P_{i}=0$. From calculus, the rate of change of $\mathrm{t} / \tau=1 / \tau$. The final equation then becomes

Rate of change of $(\ln \mathrm{P})=1 / \tau$

The power for this type of equation may be measured at any time after the reactor begins to increase power. This power is measured by an ionization chamber which produces a current proportional to the neutron flux or power. The current produced in the chamber is sent to a logarithmic amplifier, which translates this signal into a voltage proportional to the logarithm of current $i$. This voltage leads into a rate-ofchange circuit, whose output voltage is proportional to approximately $1 / \tau$. A trip circuit is attached to this channel and, if the voltage proportional to $1 / \tau$ exceeds some preset level, the circuit will open, allowing the safety rods to drop.

\subsection{Automatic Control Circuits - Regulating Rod}

The control circuit for the regulating rod senses the power level of a reactor and compares this level with a preset standard current. This comparison results in the appropriate motion of the regulating rod to keep the currents equal, thus maintaining the predetermined power level.

An automatic control system usually consists of a control loop around the regulating rod and may be operated as either a proportional regulating system or as a discontinuous regulating system. A proportional regulating system is one in which position of the regulating or control rod is changed in proportion to and in phase opposition with any error caused either by changes of power demand or by transient, internal system changes. On the other hand, a discontinuous regulating system is one in which no movement of the regulating rod occurs, unless an error, greate $r$ in magnitude than some fixed percentage, is set up within the control loop. When sufficient deviation from the demand conditions exists, the position of the regulating rod is changed at some fixed velocity. In illustration, the regulating rod at the CP-5 reactor is limited to a motion of $0.5 \mathrm{~cm} / \mathrm{sec}$.

The comparison of the two currents, ionization chamber and standard, is of great importance, and its function is twofold. First, it provides that the error signal is essentially the subtraction between the output of the neutron-detector signal and a power-demand signal; secondly, it is used to compensate for the nonlinearity of a reactor.

A simple control circuit for the regulating rod is shown in Figure 6.7. The ionization chamber produces a voltage $V_{P}$ (power voltage) across a resistor. This voltage is proportional to the power level of the reactor. 
Another voltage, the demand voltage $\mathrm{V}_{\mathrm{D}}$, is produced across a second resistor with a suitable power supply as the point of origin. The second resistor contains a variable slider device that allows adjustment for various demand voltages. Both voltages are compared in a differential amplifier which supplies power to the regulating rod motor. So, if

1. $V_{P}$ is greater than demand power $V_{D}$, the rod runs into the core, decreasing reactivity.

2. $V_{P}$ is less than $V_{D}$, the rod runs out, increasing reactivity.

3. $\mathrm{V}_{\mathrm{P}}$ is equal to $\mathrm{V}_{\mathrm{D}}$, the motion is halted.

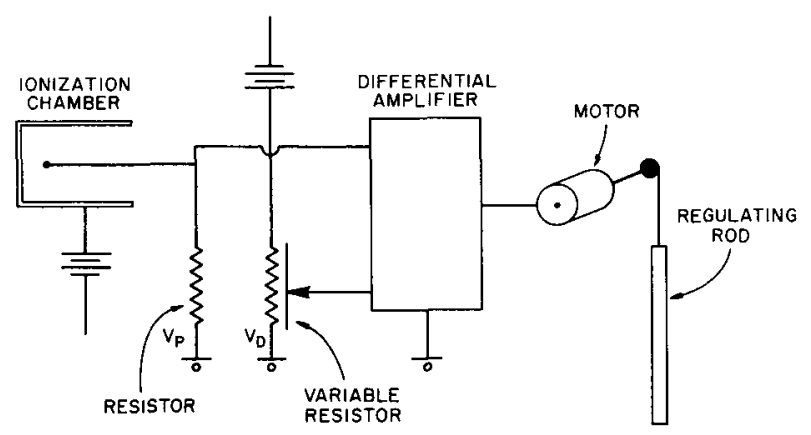

Fig. 6.7

Automatic Control Circuit

\subsection{Reliability of Safety Systems}

In closing, we might briefly discuss some of the problems of design of reactor safety circuits. The paramount factor when a scramming situation exists is that the odds of the system failing to respond must be extremely small. A corollary nuisance item is created because for reactor efficiency the number of false scrams must also be small.

We have two basic situations which must be considered in reactor design. First, the reactor with large negative temperature coefficients and small excess reactivities will help to protect itself in most emergency situations. Other types of reactors cannot depend on this built-in protection, and additional external scramming circuits must be provided. Of course, external scram circuits must be furnished in the first case, but here the reliability of the circuits does not have to be quite as great.

It should be obvious that completely failsafe circuits cannot be developed without a reactor being occasionally shut down by false scrams. One method of minimizing the number of these scrams would be to provide duplicate channel equipment of all reasonable scramming functions, and then make each channel out of the best and most reliable components. The reactor can run on one set of components while the other units are being checked out or repaired. The use of any particular scheme of safety circuits will be determined by the needs of a particular reactor. You, as 
an operator, will possibly be in a position to increase reactor economy by thoroughly learning the nuclear instrumentation of your assigned reactor.

The amplification, counting, differentiating, and integrating circuits which follow all of the detecting elements just described are complex subjects, and considerable literature is available on design and use. All of these circuits fall into two categories: pulse-counting and currentmeasuring types. The essential thing to remember from a control point of view is that the output of these circuits is always in the form of an electrical signal, which is in some manner proportional to the neutron level in the reactor. At this point, you are aware of some of the complexities in any reactor. Fundamentally the limit on the power output or operating level of the research reactor is twofold; first, the requirements of the various research groups and, more importantly that concerned with the temperature. Temperature distribution greatly depends upon the position of the control rods, and it is possible to make a reactor critical with many different combinations of control-rod configurations. We might point out that an alert operator will occasionally trim rod positions to keep the reactor always in the best possible flux configuration for the established operating conditions. Refer to this information frequently, and, as your knowledge increases, move on to further research material. Your reactor and shift supervisors are always available to answer any questions and problems you may have, regarding reactor work. 


\section{GLOSSARY}

$-A-$

ALPHA PARTICLE: A helium nucleus, consisting of two protons and two neutrons, with a double positive charge.

AMPERE: Practical unit of electric current; the flow of one coulomb/sec.

AMPLIFIER, LINEAR: A pulse amplifier in which the output pulse height is proportional to an input pulse height for a given pulse shape up to a point at which the amplifier overloads.

ANION: Negatively charged ion.

ANODE: Positive electrode; electrode to which negative ions are attracted.

ATOM: Smallest particle of an element which is capable of entering into a chemical reaction.

ATOMIC MASS: The mass of a neutral atom of a nuclide, usually expressed in terms of atomic mass units.

ATOMIC MASS UNIT (Symbol AMU): One sixteenth of the mass of one neutral atom of oxygen-16 equivalent to $1.657 \times 10^{-24} \mathrm{gm}, 931 \mathrm{Mev}$, $1.49 \times 10^{-3} \mathrm{erg}$, or 0.999728 atomic weight units.

ATOMIC NUMBER (Symbol Z): The number of orbital electrons surrounding the nucleus of a neutral atom, and the number of protons in the nucleus.

ATOMIC WEIGHT: The total weight of the nucleus and orbital electrons for one atom of a species.

ATTENUATION: The process by which a beam of radiation is reduced in intensity when passing through some material. It is the combination of absorption and scattering processes, and leads to a decrease in flux density of the bean when projected through matter.

AVOGADRO'S NUMBER $\left(6.025 \times 10^{23}\right)$ : Number of atoms in a gram atomic weight of any element; also, the number of molecules in the gram molecular weight of any substance.

BARN: Unit expressing the probability of a specific nuclear reaction taking place in terms of cross sectional area. Numerically, it is $10^{-24} \mathrm{~cm}^{2}$. 
BEAM: A unidirectional or approximately unidirectional flow of electromagnetic radiation or of particles.

BEAM HOLE: A hole through the shield and usually through the reflector of a reactor to permit the escape of a beam of radiation, in particular a beam of fast neutrons, for experimental purposes.

BETA PARTICLE: Charged particle emitted from the nucleus of an atom and having a mass and charge equal in magnitude to those of the electron.

$$
-\mathrm{C}-
$$

CALORIE (Gram calorie): Amount of heat necessary to raise the temperature of one gram of water one degree centigrade.

CAPTURE CROSS SECTION: See cross section.

CATHODE: Negative electrode; electrode to which positive ions are attracted.

CATION: Positively charged ion.

CHAMBER, IONIZATION: An instrument designed to measure quantity of ionizing radiation in terms of the charge of electricity associated with ions produced within a defined volume.

COLLISION: An encounter between two subatomic particles which changes the existing momentum and energy conditions.

COLUMN, THERMAL: A column or large body of moderator, such as graphite, extending away from the active section of a nuclear reactor to provide near its other end a flux of thermal neutrons of high cadmium ratio, i.e., containing few fast or epithermal neutrons.

COMPTON EFFECT: An attenuation process for $\mathrm{X}$ or gamma radiation in which an incident photon interacts with an orbital electron to produce a recoil electron and a scattered photon of energy less than the incident photon.

CONTROL, REACTOR: The purposeful variation of the reactivity of a reactor. Absorber control is obtained by variation of the amount of neutron absorbers within the reactor.

COOLANT: A substance, ordinarily fluid, used for cooling any part of the reactor in which heat is generated.

CORE: In a nuclear reactor, the region containing the fissionable material. 
COSMIC RAYS: High-energy particulate and electromagnetic radiations which originate outside the earth's atmosphere.

CRITICAL: Capable of sustaining (at a constant level) a chain reaction. Prompt critical is the capability of sustaining a chain reaction without the aid of delayed neutrons.

CROSS SECTION, CAPTURE: The probability that a nucleus will capture an incident particle. The unit of measure is commonly the barn $\left(10^{-24} \mathrm{~cm}^{2}\right)$.

- D-

DELAYED NEUTRON: Neutrons emitted by excited nuclei formed in a radioactive process, so-called because they are emitted an appreciable time after fission. They are important in control of nuclear reactors.

DEUTERIUM: A heavy isotope of hydrogen having one proton and one neutron in the nucleus.

ELECTRON: A negatively charged particle which is a constituent of every neutral atom. A unit of negative electricity equal to $4.8 \times 10^{-10}$ electrostatic unit or $1.6 \times 10^{-19}$ coulomb. Its mass is 0.000549 atomic mass units.

ELECTRON VOLT: A unit of energy equal to the amount of energy gained by an electron in passing through a potential difference of one volt.

ELEMENT: Pure substance, consisting of atoms of the same atomic number, which cannot be decomposed by ordinary chemical means.

ENRICHED URANIUM: Uranium in which the abundance of the $\mathrm{U}^{235}$ isotope is increased above normal.

ERG: Unit of work done by a force of one dyne acting through a distance of one $\mathrm{cm}$.

$$
-\mathrm{F}-
$$

FISSION, NUCLEAR: A nuclear transformation characterized by the splitting of a nucleus into at least two other nuclei, accompanied by the release of a relatively large amount of energy.

FISSION PRODUCTS: Elements resulting from fission.

FISSION YIELD: The percentage of fissions leading to a particular nuclide by direct formation and by decay of precursors.

FLUX: The number of photons or particles flowing per unit time across one square centimeter. 
FUEL, NUCLEAR: Fissionable material used or usable in producing energy in a nuclear reactor.

$$
-G-
$$

GAMMA, PROMPT: Gamma radiation emitted at the time of fission of a nucleus, or at the time a nucleus captures a particle.

GAMMA RAY: Electromagnetic radiation of short wavelength, with a range of wavelengths from $10^{-8}$ to $10^{-11} \mathrm{~cm}$, emitted from the nucleus.

GAS MULTIPLICATION: As applied to the gas ionization radiation detecting instruments, the ratio of the charge collected to the charge produced by the initial ionizing event.

$$
-\mathrm{H}-
$$

HALF-LIFE, RADIOACTIVE: The time required for a radioactive substance to lose $50 \%$ of its activity by decay.

$$
-\mathrm{I}-
$$

INTERLOCK: A device, usually electrical and (or) mechanical in nature, to prevent activation of a control unit until a preliminary condition has been met. Its purpose is usually safety of personnel and equipment.

ION: An atomic particle, atom, or chemical radical bearing an electrical charge, either positive or negative.

ION EXCHANGE: A chemical process involving interchange of ions between a solution and a particular solid material, such as an ion-exchange resin, consisting of a matrix of insoluble material interspersed with fixed ions of opposite charge.

IONIZATION: The process or result of any process by which a neutral atom or molecule acquires either a positive or a negative charge.

ISOTOPE: One of several nuclides having the same number of protons in their nucleus, and hence having the same atomic number, but differing in the number of neutrons, and therefore in the mass number.

$-\mathrm{M}-$

MASS NUMBER: The number of protons and neutrons in the nucleus of an atom.

MEV: The symbol for one million electron volts. 
MODERATOR: Materials used in a reactor to moderate, i.e., slow down, neutrons from the high energies at which they are released.

MOLECULAR WEIGHT: The sum of the atomic weights of all the atoms in a molecule.

MOLECULE: The quantity of a compound which can exist by itself and retain all the properties of the original substance.

$-\mathrm{N}-$

NEUTRINO: A neutral particle of very small rest mass postulated to account for the continuous distribution of energy among the particles in the betadecay process.

NEUTRON: An elementary nuclear particle with a mass approximately equal to that of a hydrogen atom and electrically neutral. Its mas is 1.008982 atomic mass units. Neutrons are commonly divided into subclassifications according to their energies as follows: thermal neutrons, around $0.025 \mathrm{ev;}$ intermediate, $10^{2}$ to $10^{5} \mathrm{ev}$; fast, greater than $0.1 \mathrm{Mev}$.

NUCLEUS: That part of an atom in which the total positive electric charge and most of the mass are concentrated.

NUCLIDE: A species of atom characterized by the constitution of its nucleus. The nuclear constitution is specified by the number of protons, $Z$, number of neutrons, $N$, and energy content; or, alternatively, by the atomic number $\mathrm{Z}$, mass number $\mathrm{A}=(\mathrm{N}+\mathrm{Z})$, and atomic mass. To be regarded as a distinct nuclide, the atom must be capable of existing for a measurable time; thus, nuclear isomers are separate nuclides, whereas promptly decaying excited nuclear states and unstable intermediates in nuclear reactions are not so considered.

$-P-$

PAIR PRODUCTION: An absorption process for gamma radiation in which the incident photon is annihilated in the vicinity of the nucleus of the absorbing atom with subsequent production of an electron and positron pair. This reaction occurs only for incident photon energies exceeding $1.02 \mathrm{Mev}$.

PERIOD: In a nuclear reactor in which the neutron flux is rising or falling exponentially, the time required for it to change by a factor of e (i.e., by $2.718)$.

PHOTOELECTRIC EFFECT: A process by which a photon ejects an electron from an atom. All the energy of the photon is absorbed in ejecting the electron and imparting kinetic energy to it. 
PHOTON: A quantity of electromagnetic energy whose value in ergs is the product of its frequency in cycles per second and Planck's constant. The equation is $\mathrm{E}=\mathrm{h} \nu$.

POSITRON: Particle equal in mass to the electron and having an equal but opposite charge.

PROTON: An elementary nuclear particle with a positive electric charge equal numerically to the charge of the electron and a mass of 1.007594 atomic mass units.

$-S-$

SCATTERING, COMPTON: The inelastic scattering of a photon through interaction with atomic electrons, accompanied by ejection of a recoil electron from the atom with which the interaction occurred. Compton-scattered photons carry away a fraction of the incident photon energy, ranging from an average of about $85 \%$ of the initial energy for a $0.1-\mathrm{Mev}$ photon to an average of about $30 \%$ for a $10-\mathrm{Mev}$ photon. 